\title{
Basins of attraction of period-two solutions of monotone difference equations
}

\author{
Arzu Bilgin', Mustafa RS Kulenović ${ }^{*}$ and Esmir Pilav²
}

"Correspondence:

mkulenovic@uri.edu

'Department of Mathematics,

University of Rhode Island, Kingston, RI 02881-0816, USA

Full list of author information is

available at the end of the article

\begin{abstract}
We investigate the global character of the difference equation of the form

$$
x_{n+1}=f\left(x_{n}, x_{n-1}\right), \quad n=0,1, \ldots
$$

with several period-two solutions, where $f$ is increasing in all its variables. We show that the boundaries of the basins of attractions of different locally asymptotically stable equilibrium solutions or period-two solutions are in fact the global stable manifolds of neighboring saddle or non-hyperbolic equilibrium solutions or period-two solutions. As an application of our results we give the global dynamics of three feasible models in population dynamics which includes the nonlinearity of Beverton-Holt and sigmoid Beverton-Holt types.
\end{abstract}

MSC: 39A10; 39A20; 37B25; 37D10

Keywords: attractivity; basin; difference equation; invariant manifolds; period-two solutions

\section{Introduction}

Let $I$ be some interval of real numbers and let $f \in C^{1}[I \times I, I]$ be such that $f(I \times I) \subseteq \mathcal{K}$ where $\mathcal{K} \subseteq I$ is a compact set. Let $\bar{x}_{0}, \bar{x}_{\mathrm{SW}}, \bar{x}_{\mathrm{NE}} \in I, \bar{x}_{0}<\bar{x}_{\mathrm{SW}}<\bar{x}_{\mathrm{NE}}$ be three equilibrium points of the difference equation

$$
x_{n+1}=f\left(x_{n}, x_{n-1}\right), \quad n=0,1, \ldots,
$$

where $f$ is a continuous and increasing function in both variables. There are several global attractivity results for equation (1) which give the sufficient conditions for all solutions to approach a unique equilibrium. We list three such results.

The first theorem, which has also been very useful in applications to mathematical biology, was really motivated by a problem in [1].

Theorem 1 (See [1] and [2], p.9) Let $I \subseteq[0, \infty)$ be some interval and assume that $f \in$ $C[I \times I,(0, \infty)]$ satisfies the following conditions:

(i) $f(x, y)$ is non-decreasing in each of its arguments; 
(ii) equation (1) has a unique positive equilibrium point $\bar{x}$ and the function $f(x, x)$ satisfies the negative feedback condition

$$
(x-\bar{x})(f(x, x)-x)<0 \quad \text { for every } x \in I-\{\bar{x}\} .
$$

Then every positive solution of equation (1) with initial conditions in I converges to $\bar{x}$.

Theorem 2 Let $[a, b]$ be an interval of real numbers and assume that

$$
f:[a, b] \times[a, b] \rightarrow[a, b]
$$

is a continuous function satisfying the following properties:

(a) $f(x, y)$ is non-decreasing in each of its arguments;

(b) equation (1) has a unique equilibrium $\bar{x} \in[a, b]$.

Then every solution of equation (1) converges to $\bar{x}$.

Theorems 1 and 2 were extended to a $k$ th order difference equation where the right hand side is a non-decreasing function of all its variables; see $[1,2]$.

The following result has been obtained in [3].

Theorem 3 Let $I \subseteq R$ and let $f \in C[I \times I, I]$ be a function which increases in both variables. Then for every solution of equation (1) the subsequences $\left\{x_{2 n}\right\}_{n=0}^{\infty}$ and $\left\{x_{2 n+1}\right\}_{n=-1}^{\infty}$ of even and odd terms of the solution do exactly one of the following:

(i) Eventually they are both monotonically increasing.

(ii) Eventually they are both monotonically decreasing.

(iii) One of them is monotonically increasing and the other is monotonically decreasing.

Remark 1 Theorem 1 is actually a special case of Theorem 2. Indeed (2) implies that there exist $a$ and $b, a<\bar{x}<b$ such that $f(a, a)>a, f(b, b)<b$, which in view of monotonicity of $f$ implies that

$$
f:[a, b] \times[a, b] \rightarrow[a, b]
$$

and so all conditions of Theorem 2 are satisfied. Furthermore, Theorem 2 is a special case of Theorem 3 if we additionally assume non-existence of period-two solutions and singular points on the boundary of the region which may be the limiting points of the solutions of equation (1). See [4] for higher order versions of Theorems 1 and 2. None of these results provide any information as regards the basins of attraction of different equilibrium solutions or period-two solutions when there exist several equilibrium solutions and periodtwo solutions. Theorem 3 has been applied to many equations, see [3,5] and references therein, but so far no example of equation (1) with a function $f$ increasing in both variables which has stable equilibrium points and stable period-two solutions has been exhibited. In this paper we give several such examples for difference equations of the type

$$
x_{n+1}=f_{0}\left(x_{n}\right)+f_{1}\left(x_{n-1}\right), \quad n=0,1, \ldots,
$$

where $f_{i}, i=0,1$ are continuous and increasing functions. Many difference equations in mathematical biology are of the form (3); see [6] and references therein. As we have shown 
in [7] if the first order difference equation $x_{n+1}=f_{1}\left(x_{n}\right)$ has two equilibrium points the corresponding delay difference equation $x_{n+1}=f_{1}\left(x_{n-1}\right)$ has one period-two solution. Now if $f_{1}$ dominates $f_{0}$ in equation (3) then one may expect equation (3) will have period-two solution as well, which we show in this paper. Similarly if the difference equation $x_{n+1}=f_{1}\left(x_{n}\right)$ has $k$ equilibrium points the corresponding delay difference equation $x_{n+1}=f_{1}\left(x_{n-1}\right)$ has $k(k-1) / 2$ period-two solutions, and if $f_{1}$ dominates $f_{0}$ in equation (3) then we show that equation (3) has a period-two solution as well.

In [5] the authors consider the difference equation (1) with several equilibrium points under the condition of the nonexistence of minimal period-two solutions and determine the basins of attraction of different equilibrium solutions. In this paper we consider equation (1) which has three equilibrium points and up to three minimal period-two solutions which are in North-East ordering. More precisely, we will give sufficient conditions for the precise description of the basins of attraction of different equilibrium points and periodtwo solutions. The results can be immediately extended to the case of any number of the equilibrium points and the period-two solutions by replicating our main results. An application of our results gives a precise description of the basis of attraction of all attractors of several difference equations, which are feasible models in population dynamics. Precisely, we illustrate our results with applications to three difference equations where all functions are linear, Beverton-Holt or sigmoid Beverton-Holt. In fact, our general results here are motivated by equations ( 8 ) and (14). Our results give first examples of difference equations with coexisting stable equilibrium solutions and stable period-two solutions.

\section{Preliminaries}

We now give some basic notions about monotone maps in the plane and connection between equation (1) and the monotone map.

Consider a map $T$ on a nonempty set $\mathcal{S} \subset \mathbb{R}^{2}$, and let $\overline{\mathbf{e}} \in \mathcal{S}$. The point $\overline{\mathbf{e}} \in \mathcal{S}$ is called a fixed point if $T(\overline{\mathbf{e}})=\overline{\mathbf{e}}$. An isolated fixed point is a fixed point that has a neighborhood with no other fixed points in it. A fixed point $\overline{\mathbf{e}} \in \mathcal{S}$ is an attractor if there exists a neighborhood $\mathcal{U}$ of $\overline{\mathbf{e}}$ such that $T^{n}(\mathbf{x}) \rightarrow \overline{\mathbf{e}}$ as $n \rightarrow \infty$ for $\mathbf{x} \in \mathcal{U}$; the basin of attraction is the set of all $\mathbf{x} \in \mathcal{S}$ such that $T^{n}(\mathbf{x}) \rightarrow \overline{\mathbf{e}}$ as $n \rightarrow \infty$. A fixed point $\overline{\mathbf{e}}$ is a global attractor on a set $\mathcal{K}$ if $\overline{\mathbf{e}}$ is an attractor and $\mathcal{K}$ is a subset of the basin of attraction of $\overline{\mathbf{e}}$. If $T$ is differentiable at a fixed point $\overline{\mathbf{e}}$, and if the Jacobian $J_{T}(\overline{\mathbf{e}})$ has one eigenvalue with modulus less than one and a second eigenvalue with modulus greater than one, $\overline{\mathbf{e}}$ is said to be a saddle. If one of the eigenvalues of $T$ has absolute values 1 and a second eigenvalue has modulus greater (resp. less) than one, then $\overline{\mathbf{e}}$ is said to be a non-hyperbolic of stable (resp. unstable) type. See [8] for additional definitions (stable and unstable manifolds, asymptotic stability).

Consider a partial ordering $\preceq$ on $\mathbb{R}^{2}$. Two points $\mathbf{v}, \mathbf{w} \in \mathbb{R}^{2}$ are said to be related if $\mathbf{v} \preceq \mathbf{w}$ or $\mathbf{w} \preceq \mathbf{v}$. Also, a strict inequality between points may be defined as $\mathbf{v} \prec \mathbf{w}$ if $\mathbf{v} \preceq \mathbf{w}$ and $\mathbf{v} \neq \mathbf{w}$. A stronger inequality may be defined as $\mathbf{v}=\left(v_{1}, v_{2}\right) \ll \mathbf{w}=\left(w_{1}, w_{2}\right)$ if $\mathbf{v} \preceq \mathbf{w}$ with $v_{1} \neq w_{1}$ and $v_{2} \neq w_{2}$. For $\mathbf{u}, \mathbf{v}$ in $\mathbb{R}^{2}$, the order interval $\llbracket \mathbf{u}, \mathbf{v} \rrbracket$ is the set of all $\mathbf{x} \in \mathbb{R}^{2}$ such that $\mathbf{u} \preceq \mathbf{x} \preceq \mathbf{v}$.

A map $T$ on a nonempty set $\mathcal{S} \subset \mathbb{R}^{2}$ is a continuous function $T: \mathcal{S} \rightarrow \mathcal{S}$. The map $T$ is monotone if $\mathbf{v} \preceq \mathbf{w}$ implies $T(\mathbf{v}) \preceq T(\mathbf{w})$ for all $\mathbf{v}, \mathbf{w} \in \mathcal{S}$, and it is strongly monotone on $\mathcal{S}$ if $\mathbf{v} \prec \mathbf{w}$ implies that $T(\mathbf{v}) \ll T(\mathbf{w})$ for all $\mathbf{v}, \mathbf{w} \in \mathcal{S}$. The map is strictly monotone on $\mathcal{S}$ if $\mathbf{v} \prec \mathbf{w}$ implies that $T(\mathbf{v}) \prec T(\mathbf{w})$ for all $\mathbf{v}, \mathbf{w} \in \mathcal{S}$. Clearly, being related is invariant under iteration of a strongly monotone map. 
Throughout this paper we shall use the North-East ordering for which the positive cone is the first quadrant, i.e. this partial ordering is defined by

$$
\left(x_{1}, y_{1}\right) \preceq_{\mathrm{ne}}\left(x_{2}, y_{2}\right) \quad \Leftrightarrow \quad x_{1} \leq x_{2} \quad \text { and } \quad y_{1} \leq y_{2} .
$$

A map $T$ on a nonempty set $\mathcal{S} \subset \mathbb{R}^{2}$ which is monotone with respect to the NorthEast ordering is called cooperative and a map monotone with respect to the South-East ordering

$$
\left(x_{1}, y_{1}\right) \preceq_{\text {se }}\left(x_{2}, y_{2}\right) \quad \Leftrightarrow \quad x_{1} \leq x_{2} \quad \text { and } \quad y_{1} \geq y_{2}
$$

is called competitive.

If $T$ is differentiable map on a nonempty set $\mathcal{S}$, a sufficient condition for $T$ to be strongly monotonic with respect to the NE ordering is that the Jacobian matrix at all points $\mathbf{x}$ has the sign configuration

$$
\operatorname{sign}\left(J_{T}(\mathbf{x})\right)=\left[\begin{array}{ll}
+ & + \\
+ & +
\end{array}\right],
$$

provided that $\mathcal{S}$ is open and convex.

For $\mathbf{x} \in \mathbb{R}^{2}$, define $Q_{\ell}(\mathbf{x})$ for $\ell=1, \ldots, 4$ to be the usual four quadrants based at $\mathbf{x}$ and numbered in a counterclockwise direction, for example, $Q_{1}(\mathbf{x})=\left\{\mathbf{y} \in \mathbb{R}^{2}: x_{1} \leq y_{1}, x_{2} \leq y_{2}\right\}$. The (open) ball of radius $r$ centered at $\mathbf{x}$ is denoted with $\mathcal{B}(\mathbf{x}, r)$. If $\mathcal{K} \subset \mathbb{R}^{2}$ and $r>0$, write $\mathcal{K}+\mathcal{B}(\mathbf{0}, r):=\{\mathbf{x}: \mathbf{x}=\mathbf{k}+\mathbf{y}$ for some $\mathbf{k} \in \mathcal{K}$ and $\mathbf{y} \in \mathcal{B}(\mathbf{0}, r)\}$. If $\mathbf{x} \in[-\infty, \infty]^{2}$ is such that $\mathbf{x} \preceq \mathbf{y}$ for every $\mathbf{y}$ in a set $\mathcal{Y}$, we write $\mathbf{x} \preceq \mathcal{Y}$. The inequality $\mathcal{Y} \preceq \mathbf{x}$ is defined similarly.

The next result in [9] is stated for order-preserving maps on $\mathbb{R}^{n}$ and it also holds in ordered Banach spaces [10].

Theorem 4 For a nonempty set $R \subset \mathbb{R}^{n}$ and $\leq$ a partial order on $\mathbb{R}^{n}$, let $T: R \rightarrow R$ be an order-preserving map, and let $a, b \in R$ be such that $a \prec b$ and $\llbracket a, b \rrbracket \subset R$. If $a \preceq T(a)$ and $T(b) \preceq b$, then $\llbracket a, b \rrbracket$ is an invariant set and:

i. There exists a fixed point of $T$ in $\llbracket a, b \rrbracket$.

ii. If $T$ is strongly order preserving, then there exists a fixed point in $\llbracket a, b \rrbracket$ which is stable relative to $\llbracket a, b \rrbracket$.

iii. If there is only one fixed point in $\llbracket a, b \rrbracket$, then it is a global attractor in $\llbracket a, b \rrbracket$ and therefore asymptotically stable relative to $\llbracket a, b \rrbracket$.

The following result is a direct consequence of the trichotomy theorem of Dancer and Hess; see $[9,10]$.

Corollary 1 If the non-negative cone of a partial ordering $\preceq$ is a generalized quadrant in $\mathbb{R}^{n}$, and if $T$ has no fixed points in $\llbracket u_{1}, u_{2} \rrbracket$ other than $u_{1}$ and $u_{2}$, then the interior of $\llbracket u_{1}, u_{2} \rrbracket$ is either a subset of the basin of attraction of $u_{1}$ or a subset of the basin of attraction of $u_{2}$.

We say that $f$ is strongly increasing in both arguments if it is increasing, differentiable, and has both partial derivatives positive in a considered set. The connection between the theory of monotone maps and the asymptotic behavior of equation (1) follows from the 
fact that if $f$ is strongly increasing, then a map associated to equation (1) is a cooperative map on $\mathcal{I} \times \mathcal{I}$ while the second iterate of a map associated to equation (1) is a strictly cooperative map on $\mathcal{I} \times \mathcal{I}$.

Set $x_{n-1}=u_{n}$ and $x_{n}=v_{n}$ in equation (1) to obtain the equivalent system

$$
\begin{aligned}
& u_{n+1}=v_{n}, \\
& v_{n+1}=f\left(v_{n}, u_{n}\right),
\end{aligned} \quad n=0,1, \ldots .
$$

Let $F(u, v)=(v, f(v, u))$. Then $F$ maps $I \times I$ into itself and is a cooperative map. The second iterate $T:=F^{2}$ is given by

$$
T(u, v)=(f(v, u), f(f(v, u), v))
$$

and it is clearly strictly cooperative on $\mathcal{I} \times \mathcal{I}$.

If $D_{1} g(u, v)$ and $D_{2} g(u, v)$ denote the partial derivatives of a function $g(u, v)$ with respect to $u$ and $v$, the Jacobian matrix of $T$ is

$$
J_{T}(u, v)=\left(\begin{array}{cc}
D_{2} f(v, u) & D_{1} f(v, u) \\
D_{1} f(f(v, u), v) D_{2} f(v, u) & D_{1} f(f(v, u), v) D_{1} f(v, u) \\
& +D_{2} f(f(v, u), v)
\end{array}\right) .
$$

The determinant of (7) is given by

$$
\operatorname{det} J_{T}(u, v)=D_{2} f(v, u) D_{2} f(f(v, u), v)>0
$$

To check injectivity of $T$ we set

$$
T\left(\left(u_{1}, v_{1}\right)\right)=T\left(\left(u_{2}, v_{2}\right)\right),
$$

which implies

$$
f\left(v_{1}, u_{1}\right)=f\left(v_{2}, u_{2}\right), \quad f\left(f\left(v_{1}, u_{1}\right), v_{1}\right)=f\left(f\left(v_{2}, u_{2}\right), v_{2}\right)
$$

and so $f\left(f\left(v_{1}, u_{1}\right), v_{1}\right)=f\left(f\left(v_{1}, u_{1}\right), v_{2}\right)$. By using the monotonicity of $f$ we conclude that $v_{1}=v_{2}$ which in view of $f\left(v_{1}, u_{1}\right)=f\left(v_{2}, u_{2}\right)$ gives $u_{1}=u_{2}$.

The theory of monotone maps has been extensively developed at the level of ordered Banach spaces and applied to many types of equations such as ordinary, partial, and discrete types; see [10-16]. In particular, [12] has an extensive updated bibliography of different aspects of the theory of monotone maps. The theory of monotone discrete maps is more specialized and so one should expect stronger results in this case. An excellent review of the basic results is given in $[12,13]$. In particular, two-dimensional discrete maps are studied in great detail and very precise results which describe the global dynamics and the basins of attractions of equilibrium points and period-two solutions as well as global stable manifolds are given in [11, 14, 17-20].

\section{Main results}

Let $I$ be some interval of real numbers and let $f \in C^{1}[I \times I, I]$ be strongly increasing function. Assume that for $\left(x_{0}, y_{0}\right) \in I \times I$ there exists $n_{0}$ such that $F^{n}\left(x_{0}, y_{0}\right) \in\left[U_{1}, U_{2}\right]^{2}$ 
for all $n>n_{0}$ where $\left[U_{1}, U_{2}\right] \subseteq I$ and $-\infty<U_{1}<U_{2}<\infty$ and assume that $\left[U_{1}, U_{2}\right]^{2}$ is an invariant set for the map $T$, that is, $T:\left[U_{1}, U_{2}\right]^{2} \rightarrow\left[U_{1}, U_{2}\right]^{2}$. Let $\bar{x}_{0}, \bar{x}_{\mathrm{SW}}, \bar{x}_{\mathrm{NE}} \in I$, $U_{1} \leq \bar{x}_{0}<\bar{x}_{\mathrm{SW}}<\bar{x}_{\mathrm{NE}}$ be three equilibrium points of the difference equation (1) where the equilibrium points $\bar{x}_{0}$ and $\bar{x}_{\mathrm{NE}}$ are locally asymptotically stable and $\bar{x}_{\mathrm{SW}}$ is unstable. Then the map $F$ has three equilibrium solutions $E_{0}\left(\bar{x}_{0}, \bar{x}_{0}\right), E_{\mathrm{SW}}\left(\bar{x}_{\mathrm{SW}}, \bar{x}_{\mathrm{SW}}\right)$, and $E_{\mathrm{NE}}\left(\bar{x}_{\mathrm{NE}}, \bar{x}_{\mathrm{NE}}\right)$ such that $E_{0} \ll_{\text {ne }} E_{\mathrm{SW}} \ll_{\mathrm{ne}} E_{\mathrm{NE}}$ where the equilibrium points $E_{0}$ and $E_{\mathrm{NE}}$ are locally asymptotically stable and $E_{\mathrm{SW}}$ is unstable. By Theorem 3 all solutions converge to either equilibrium solutions or to the period-two solutions. As is well known [12, 13] the periodtwo solutions are the points in the South-East ordering, which means that they belong to $Q_{2}\left(E_{S W}\right) \cup Q_{4}\left(E_{S W}\right)$. In the following discussion, we will assume that all period-two solutions belong to $\operatorname{int}\left(Q_{2}\left(E_{\mathrm{SW}}\right) \cup Q_{4}\left(E_{\mathrm{SW}}\right)\right)$.

Let $\mathcal{B}\left(E_{0}\right)$ be the basin of attraction of $E_{0}$ and $\mathcal{B}\left(E_{\mathrm{NE}}\right)$ be the basin of attraction of $E_{\mathrm{NE}}$ with respect to the map $T$.

Lemma 1 Let $Q_{1}\left(\left(x_{0}, y_{0}\right)\right)=\left\{(x, y): x \geq x_{0}\right.$ and $\left.y \geq y_{0}\right\} \cap I \times I$ and $Q_{3}\left(\left(x_{0}, y_{0}\right)\right)=\{(x, y): x \leq$ $x_{0}$ and $\left.y \leq y_{0}\right\} \cap I \times I$. Then the following holds:

(i) If there are no minimal period-two solutions in int $\left(Q_{3}\left(E_{\mathrm{SW}}\right)\right)$ then $\operatorname{int}\left(Q_{3}\left(E_{\mathrm{SW}}\right)\right) \subset \mathcal{B}\left(E_{0}\right)$.

(ii) If there are no minimal period-two solutions in $\operatorname{int}\left(Q_{1}\left(E_{\mathrm{SW}}\right)\right)$ then $\operatorname{int}\left(Q_{1}\left(E_{\mathrm{SW}}\right)\right) \subset \mathcal{B}\left(E_{\mathrm{NE}}\right)$.

Proof First, in view of Corollary 1 , int $\llbracket\left(U_{1}, U_{1}\right), E_{0} \rrbracket \subset \mathcal{B}\left(E_{0}\right)$ and also int $\llbracket E_{\mathrm{NE}},\left(U_{2}, U_{2}\right) \rrbracket \subset$ $\mathcal{B}\left(E_{\mathrm{NE}}\right)$. By Corollary 1 we obtain $\operatorname{int}\left(Q_{3}\left(E_{\mathrm{SW}}\right) \cap Q_{1}\left(E_{0}\right)\right) \subset \mathcal{B}\left(E_{0}\right)$ and $\operatorname{int}\left(Q_{1}\left(E_{\mathrm{SW}}\right) \cap\right.$ $\left.Q_{3}\left(E_{\mathrm{NE}}\right)\right) \subset \mathcal{B}\left(E_{\mathrm{NE}}\right)$. Since $\left(U_{1}, U_{1}\right) \preceq_{\text {ne }} T\left(U_{1}, U_{1}\right) \preceq_{\text {ne }} E_{0}$ and $T$ is cooperative map we obtain $T^{n}\left(U_{1}, U_{1}\right) \rightarrow E_{0}$ as $n \rightarrow \infty$. For $\left(x_{0}, y_{0}\right) \in \operatorname{int}\left(Q_{3}\left(E_{0}\right)\right)$ we have $\left(U_{1}, U_{1}\right) \preceq_{\text {ne }}$ $\left(x_{-1}, x_{0}\right) \preceq_{\text {ne }} E_{0}$, which implies $T^{n}\left(x_{-1}, x_{0}\right) \rightarrow E_{0}$ as $n \rightarrow \infty$, i.e. $\operatorname{int}\left(Q_{3}\left(E_{0}\right)\right) \subset \mathcal{B}\left(E_{0}\right)$. Assume that $\left(x_{0}, y_{0}\right) \in \operatorname{int}\left(Q_{3}\left(E_{S W}\right)\right)$. Then there exists $\left(\tilde{x}_{0}, \tilde{y}_{0}\right) \in \operatorname{int}\left(Q_{3}\left(E_{0}\right)\right)$ such that $\left(\tilde{x}_{0}, \tilde{y}_{0}\right) \preceq_{\text {ne }}\left(x_{0}, y_{0}\right)$ and $\left(\tilde{x}_{1}, \tilde{y}_{1}\right) \in \operatorname{int}\left(Q_{3}\left(E_{\mathrm{SW}}\right) \cap Q_{1}\left(E_{0}\right)\right)$ such that $\left(x_{0}, y_{0}\right) \preceq_{\text {ne }}\left(\tilde{x}_{1}, \tilde{y}_{1}\right)$. By monotonicity of $T$ we have $T^{n}\left(\tilde{x}_{0}, \tilde{y}_{0}\right) \preceq_{\text {ne }} T^{n}\left(x_{0}, y_{0}\right) \preceq_{\text {ne }} T^{n}\left(\tilde{x}_{1}, \tilde{y}_{1}\right)$, which implies $T^{n}\left(x_{0}, y_{0}\right) \rightarrow E_{0}$ as $n \rightarrow \infty$. This implies that $\operatorname{int}\left(Q_{3}\left(E_{\mathrm{SW}}\right)\right) \subset \mathcal{B}\left(E_{0}\right)$. The proof of (ii) is similar and we skip it.

Let $\mathcal{C}_{1}^{+}$denote the boundary of $\mathcal{B}\left(E_{0}\right)$ considered as a subset of $Q_{2}\left(E_{\mathrm{SW}}\right)$ (the second quadrant relative to $\left.E_{\mathrm{SW}}\right)$ and $\mathcal{C}_{1}^{-}$denote the boundary of $\mathcal{B}\left(E_{0}\right)$ considered as a subset of $Q_{4}\left(E_{\mathrm{SW}}\right)$ (the fourth quadrant relative to $\left.E_{\mathrm{SW}}\right)$. Also, let $\mathcal{C}_{2}^{+}$denote the boundary of $\mathcal{B}\left(E_{\mathrm{NE}}\right)$ considered as a subset of $Q_{2}\left(E_{\mathrm{SW}}\right)$ and $\mathcal{C}_{2}^{-}$denote the boundary of $\mathcal{B}\left(E_{0}\right)$ considered as a subset of $Q_{4}\left(E_{\mathrm{SW}}\right)$. It is easy to see that $E_{\mathrm{SW}} \in \mathcal{C}_{1}^{+}, E_{\mathrm{SW}} \in \mathcal{C}_{1}^{-}, E_{\mathrm{SW}} \in \mathcal{C}_{2}^{+}, E_{\mathrm{SW}} \in \mathcal{C}_{2}^{-}$.

The proof of the following lemma for a cooperative map is the same as the proof of Claims 1 and 2 in [21] for competitive maps, so we skip it (see Figure 1).

Lemma 2 Let $\mathcal{C}_{1}^{+}$and $\mathcal{C}_{1}^{-}$be the sets defined above. Then the sets $\mathcal{C}_{1}^{+} \cup \mathcal{C}_{1}^{-}$and $\mathcal{C}_{2}^{+} \cup \mathcal{C}_{2}^{-}$ are invariant under the map $T$ and they are the graphs of continuous strictly decreasing functions.

By Lemma 2 it remains to determine the behavior of the orbits of initial conditions $\left(x_{0}, y_{0}\right)$ such that $\left(\tilde{x}_{0}, \tilde{y}_{0}\right) \preceq$ ne $\left(x_{0}, y_{0}\right) \preceq\left(\bar{x}_{0}, \bar{y}_{0}\right)$ for some $\left(\tilde{x}_{0}, \tilde{y}_{0}\right) \in \mathcal{C}_{1}^{+} \cup \mathcal{C}_{1}^{-}$and $\left(\bar{x}_{0}, \bar{y}_{0}\right) \in$ $\mathcal{C}_{2}^{+} \cup \mathcal{C}_{2}^{-}$. 
Figure 1 Visual illustration of Lemma 2.

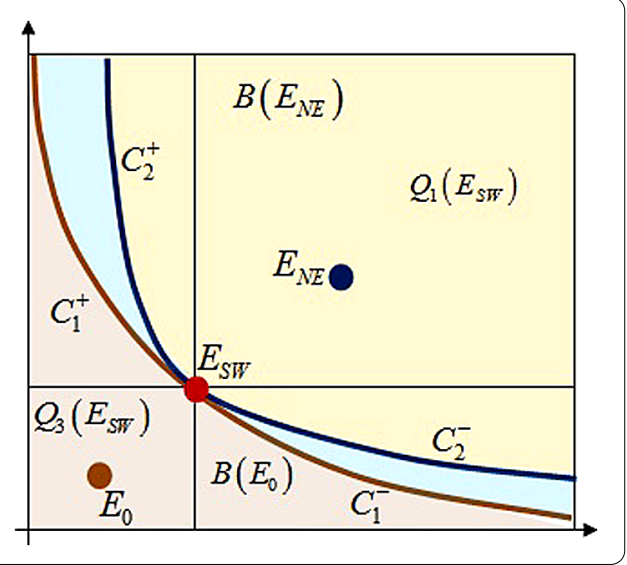

Now, we present the global dynamics of equation (1) depending on the existence or nonexistence of period-two solutions.

Theorem 5 Assume that equation (1) has no minimal period-two solutions and has three equilibrium points $\bar{x}_{0}<\bar{x}_{\mathrm{SW}}<\bar{x}_{\mathrm{NE}}$ where the equilibrium points $\bar{x}_{0}$ and $\bar{x}_{\mathrm{NE}}$ are locally asymptotically stable and $\bar{x}_{\mathrm{SW}}$ is a saddle point. Then there exist two continuous curves $\mathcal{W}^{s}\left(E_{\mathrm{SW}}\right)$ and $\mathcal{W}^{u}\left(E_{\mathrm{SW}}\right)$, both passing through the point $E_{\mathrm{SW}}$, such that $\mathcal{W}^{s}\left(E_{\mathrm{SW}}\right)$ is a graph of decreasing function and $\mathcal{W}^{u}\left(E_{\mathrm{SW}}\right)$ is a graph of an increasing function. The set of initial conditions $Q_{1}=\left\{\left(x_{-1}, x_{0}\right): x_{-1} \geq U_{1}, x_{0} \geq U_{1}\right\} \cap I$ is the union of three disjoint basins of attraction, namely $Q_{1}=\mathcal{B}\left(E_{0}\right) \cup \mathcal{B}\left(E_{\mathrm{SW}}\right) \cup \mathcal{B}\left(E_{\mathrm{NE}}\right)$, where $\mathcal{B}\left(E_{\mathrm{SW}}\right)=\mathcal{W}^{s}\left(E_{\mathrm{SW}}\right)$ and

$$
\begin{aligned}
& \mathcal{B}\left(E_{0}\right)=\left\{\left(x_{-1}, x_{0}\right) \mid\left(x_{-1}, x_{0}\right) \preceq_{\mathrm{ne}}\left(x_{E_{0}}, y_{E_{0}}\right) \text { for some }\left(x_{E_{0}}, y_{E_{0}}\right) \in \mathcal{W}^{s}\left(E_{\mathrm{SW}}\right)\right\} \\
& \mathcal{B}\left(E_{\mathrm{NE}}\right)=\left\{\left(x_{-1}, x_{0}\right) \mid\left(x_{E_{\mathrm{NE}}}, y_{E_{\mathrm{NE}}}\right) \preceq_{\mathrm{ne}}\left(x_{-1}, x_{0}\right) \text { for some }\left(x_{E_{\mathrm{NE}}}, y_{E_{\mathrm{NE}}}\right) \in \mathcal{W}^{s}\left(E_{\mathrm{SW}}\right)\right\} .
\end{aligned}
$$

Thus, we have $\mathcal{W}^{s}\left(E_{\mathrm{SW}}\right)=\mathcal{C}_{1}^{+} \cup \mathcal{C}_{1}^{-}=\mathcal{C}_{2}^{+} \cup \mathcal{C}_{2}^{-}$.

Proof By assumption the map $T$ has three equilibrium point namely $E_{0}, E_{\mathrm{SW}}$, and $E_{\mathrm{NE}}$ such that $E_{0} \ll_{\text {ne }} E_{\mathrm{SW}} \ll_{\mathrm{ne}} E_{\mathrm{NE}}$. In this case, $E_{0}$ and $E_{\mathrm{NE}}$ are locally asymptotically stable and $E_{\mathrm{SW}}$ is a saddle point. Since $f$ is strongly increasing then the map $T$ is strongly cooperative on $\left[U_{1}, \infty\right)^{2}$. It follows from the Perron-Frobenius theorem and a change of variables [14] that, at each point, the Jacobian matrix of a strongly cooperative map has two real and distinct eigenvalues, the larger one in absolute value being positive, and that corresponding eigenvectors may be chosen to point in the direction of the second and first quadrant, respectively. Also, one can show that if the map is strongly cooperative then no eigenvector is aligned with a coordinate axis.

Hence, all conditions of Theorems 1 and 4 in [19] for cooperative map $T$ are satisfied, which yields the existence of the global stable manifold $\mathcal{W}^{s}\left(E_{\mathrm{SW}}\right)$ and the global unstable manifold $\mathcal{W}^{u}\left(E_{\mathrm{SW}}\right)$, where $\mathcal{W}^{s}\left(E_{\mathrm{SW}}\right)$ is passing through the point $E_{\mathrm{SW}}$, and it is a graph of a decreasing function. The curve $\mathcal{W}^{u}\left(E_{\mathrm{SW}}\right)$ is the graph of an increasing function. Let

$$
\begin{aligned}
& \mathcal{W}^{-}=\left\{\left(x_{-1}, x_{0}\right) \mid\left(x_{-1}, x_{0}\right) \preceq_{\mathrm{ne}}\left(x_{E_{0}}, y_{E_{0}}\right) \text { for some }\left(x_{E_{0}}, y_{E_{0}}\right) \in \mathcal{W}^{s}\left(E_{\mathrm{SW}}\right)\right\}, \\
& \mathcal{W}^{+}=\left\{\left(x_{-1}, x_{0}\right) \mid\left(x_{E_{\mathrm{NE}}}, y_{E_{\mathrm{NE}}}\right) \preceq_{\mathrm{ne}}\left(x_{-1}, x_{0}\right) \text { for some }\left(x_{E_{\mathrm{NE}}}, y_{E_{\mathrm{NE}}}\right) \in \mathcal{W}^{s}\left(E_{\mathrm{SW}}\right)\right\} .
\end{aligned}
$$




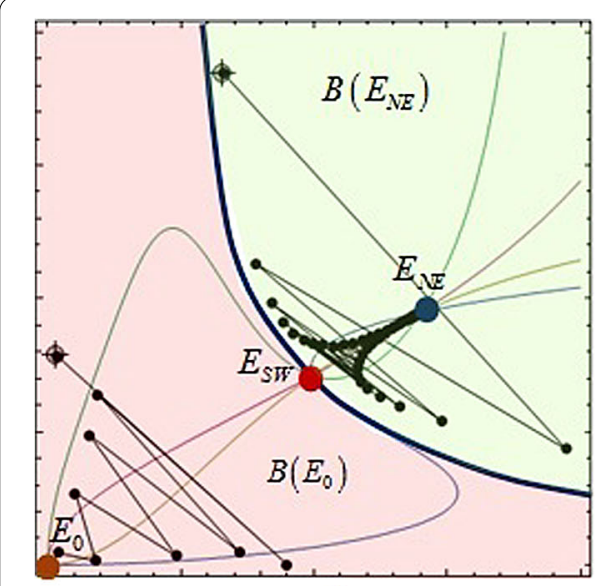

a)

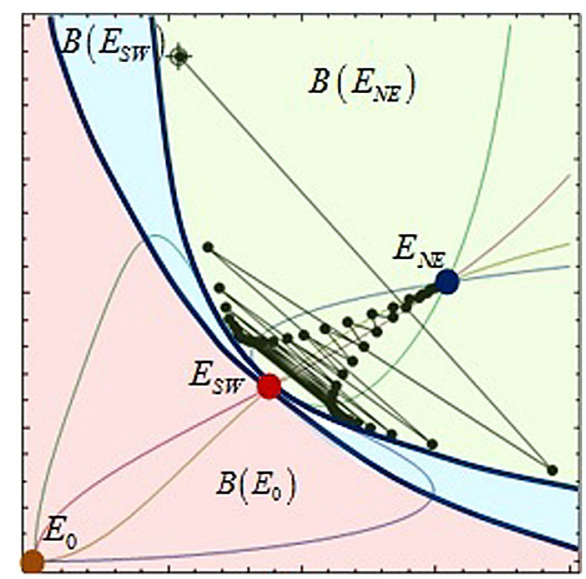

b)

Figure 2 Visual illustration of (a) Theorem 5 and (b) Theorem 6.

Take $\left(x_{0}, y_{0}\right) \in \mathcal{W}^{-} \cap\left[U_{1}, \infty\right)^{2}$ and $\left(\tilde{x}_{0}, \tilde{y}_{0}\right) \in \mathcal{W}^{+} \cap\left[U_{1}, \infty\right)^{2}$. By Theorem 4 in [19] we see that there exists $n_{0}>0$ such that $T^{n}\left(x_{0}, y_{0}\right) \in \operatorname{int}\left(Q_{3}\left(E_{\mathrm{SW}}\right)\right)$ and $T^{n}\left(\tilde{x}_{0}, \tilde{y}_{0}\right) \in \operatorname{int}\left(Q_{1}\left(E_{\mathrm{SW}}\right)\right)$ for $n>n_{0}$. The rest of the proof follows from Lemma 1. See Figure 2(a) for its visual illustration.

Theorem 6 Assume that equation (1) has no minimal period-two solutions and there exist three equilibrium points $\bar{x}_{0}<\bar{x}_{\mathrm{SW}}<\bar{x}_{\mathrm{NE}}$ where the equilibrium points $\bar{x}_{0}$ and $\bar{x}_{\mathrm{NE}}$ are locally asymptotically stable and $\bar{x}_{\mathrm{SW}}$ is non-hyperbolic. Then there exist two continuous curves $\mathcal{C}_{1}\left(E_{\mathrm{SW}}\right)$ and $\mathcal{C}_{2}\left(E_{\mathrm{SW}}\right)$ passing through the point $E_{\mathrm{SW}}$, which are the graphs of decreasing functions. The set of the initial condition $Q_{1}=\left\{\left(x_{-1}, x_{0}\right)\right\}$ is the union of three disjoint basins of attraction, namely $Q_{1}=\mathcal{B}\left(E_{0}\right) \cup \mathcal{B}\left(E_{\mathrm{SW}}\right) \cup \mathcal{B}\left(E_{\mathrm{NE}}\right)$, where

$$
\begin{aligned}
\mathcal{B}\left(E_{0}\right)=\{ & \left.\left(x_{0}, y_{0}\right) \mid\left(x_{0}, y_{0}\right) \preceq_{\mathrm{ne}}\left(x_{E_{0}}, y_{E_{0}}\right) \text { for some }\left(x_{E_{0}}, y_{E_{0}}\right) \in \mathcal{C}_{1}\left(E_{\mathrm{SW}}\right)\right\}, \\
\mathcal{B}\left(E_{\mathrm{SW}}\right)= & \left\{\left(x_{0}, y_{0}\right) \mid\left(x_{E_{0}}, y_{E_{0}}\right) \preceq_{\mathrm{ne}}\left(x_{0}, y_{0}\right) \preceq_{\mathrm{ne}}\left(x_{E_{\mathrm{NE}}}, y_{E_{\mathrm{NE}}}\right)\right. \text { for some } \\
& \left.\left(x_{E_{0}}, y_{E_{0}}\right) \in \mathcal{C}_{1}\left(E_{\mathrm{SW}}\right) \text { and }\left(x_{E_{\mathrm{NE}}}, y_{E_{\mathrm{NE}}}\right) \in \mathcal{C}_{2}\left(E_{\mathrm{SW}}\right)\right\}, \\
\mathcal{B}\left(E_{\mathrm{NE}}\right)= & \left\{\left(x_{0}, y_{0}\right) \mid\left(x_{E_{\mathrm{NE}}}, y_{E_{\mathrm{NE}}}\right) \preceq_{\mathrm{ne}}\left(x_{0}, y_{0}\right) \text { for some }\left(x_{E_{\mathrm{NE}}}, y_{E_{\mathrm{NE}}}\right) \in \mathcal{C}_{2}\left(E_{\mathrm{SW}}\right)\right\} .
\end{aligned}
$$

Proof The characterization of $\mathcal{B}\left(E_{0}\right)$ and $\mathcal{B}\left(E_{\mathrm{NE}}\right)$ follows as in Theorem 5.

The existence of the curves $\mathcal{C}_{1}\left(E_{\mathrm{SW}}\right)$ and $\mathcal{C}_{2}\left(E_{\mathrm{SW}}\right)$ passing through the point $E_{\mathrm{SW}}$ which are the graphs of decreasing functions follows from Lemma 2. Assume that $\left(x_{E_{0}}, y_{E_{0}}\right) \in$ $\mathcal{C}_{1}\left(E_{\mathrm{SW}}\right)$. Since $\mathcal{C}_{1}\left(E_{\mathrm{SW}}\right)$ is an invariant set and there are no period-two solutions we must have $T^{n}\left(x_{E_{0}}, y_{E_{0}}\right) \rightarrow E_{\mathrm{SW}}$ as $n \rightarrow \infty$. Similarly, we obtain $T^{n}\left(x_{E_{\mathrm{NE}}}, y_{E_{\mathrm{NE}}}\right) \rightarrow E_{\mathrm{SW}}$ as $n \rightarrow \infty$ if $\left(x_{E_{\mathrm{NE}}}, y_{E_{\mathrm{NE}}}\right) \in \mathcal{C}_{2}\left(E_{\mathrm{SW}}\right)$. Suppose that $\left(x_{E_{0}}, y_{E_{0}}\right) \preceq_{\text {ne }}\left(x_{0}, y_{0}\right) \preceq_{\text {ne }}\left(x_{E_{\mathrm{NE}}}, y_{E_{\mathrm{NE}}}\right)$ for some $\left(x_{E_{0}}, y_{E_{0}}\right) \in \mathcal{C}_{1}\left(E_{\mathrm{SW}}\right)$ and $\left(x_{E_{\mathrm{NE}}}, y_{E_{\mathrm{NE}}}\right) \in \mathcal{C}_{2}\left(E_{\mathrm{SW}}\right)$. Then $T^{n}\left(x_{E_{0}}, y_{E_{0}}\right) \preceq_{\text {ne }} T^{n}\left(x_{0}, y_{0}\right) \preceq_{\text {ne }}$ $T^{n}\left(x_{E_{\mathrm{NE}}}, y_{E_{\mathrm{NE}}}\right)$, which implies that $T^{n}\left(x_{0}, y_{0}\right) \rightarrow E_{\mathrm{SW}}$ as $n \rightarrow \infty$. See Figure 2(b) for the visual illustration of this result.

Now, we consider the dynamical scenarios when there exists a minimal period-two solution which is a saddle point (see Figure 3(a)). 


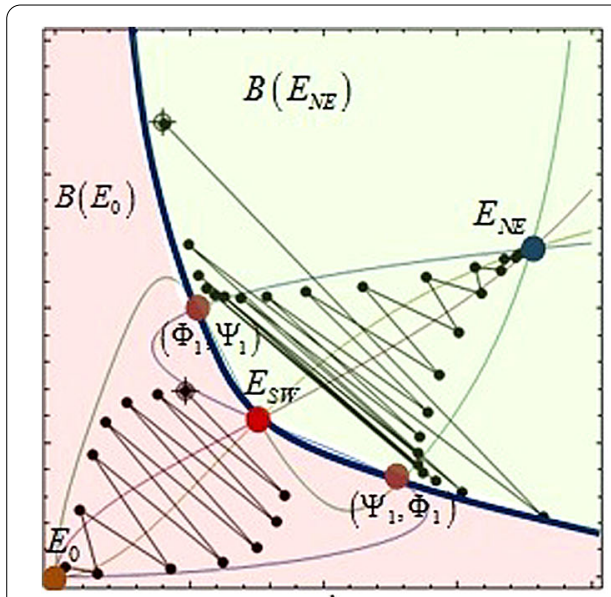

a)

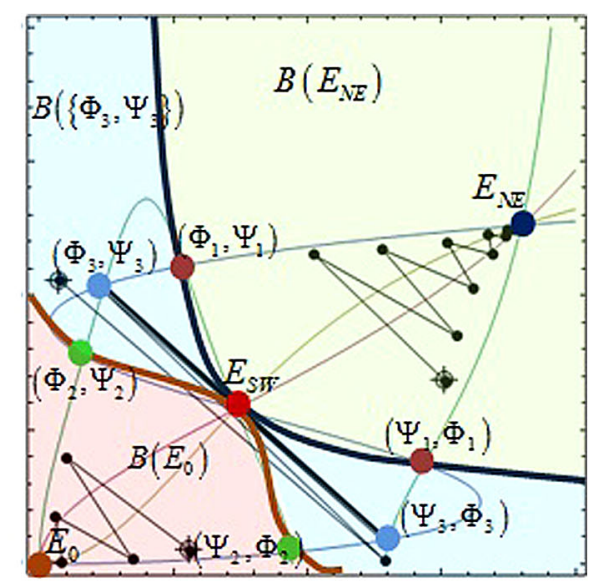

b)

Figure 3 Visual illustration of (a) Theorem 7 and (b) Theorem 8.

Theorem 7 Assume that equation (1) has three equilibrium points $U_{1} \leq \bar{x}_{0}<\bar{x}_{\mathrm{SW}}<\bar{x}_{\mathrm{NE}}$ where the equilibrium points $\bar{x}_{0}$ and $\bar{x}_{\mathrm{NE}}$ are locally asymptotically stable. Further, assume that there exists a minimal period-two solution $\left\{\Phi_{1}, \Psi_{1}\right\}$ which is a saddle point such that $\left(\Phi_{1}, \Psi_{1}\right) \in \operatorname{int}\left(Q_{2}\left(E_{S W}\right)\right)$. In this case there exist four continuous curves $\mathcal{W}^{s}\left(\Phi_{1}, \Psi_{1}\right)$, $\mathcal{W}^{s}\left(\Psi_{1}, \Phi_{1}\right), \mathcal{W}^{u}\left(\Phi_{1}, \Psi_{1}\right), \mathcal{W}^{u}\left(\Psi_{1}, \Phi_{1}\right)$, where $\mathcal{W}^{s}\left(\Phi_{1}, \Psi_{1}\right), \mathcal{W}^{s}\left(\Psi_{1}, \Phi_{1}\right)$ are passing through the point $E_{\mathrm{SW}}$ and are graphs of decreasing functions. The curves $\mathcal{W}^{u}\left(\Phi_{1}, \Psi_{1}\right), \mathcal{W}^{u}\left(\Psi_{1}, \Phi_{1}\right)$ are the graphs of increasing functions and are starting at $E_{0}$. Every solution which starts below $\mathcal{W}^{s}\left(\Phi_{1}, \Psi_{1}\right) \cup \mathcal{W}^{s}\left(\Psi_{1}, \Phi_{1}\right)$ in the North-East ordering converges to $E_{0}$ and every solution which starts above $\mathcal{W}^{s}\left(\Phi_{1}, \Psi_{1}\right) \cup \mathcal{W}^{s}\left(\Psi_{1}, \Phi_{1}\right)$ in the North-East ordering converges to $E_{\mathrm{NE}}$, i.e. $\mathcal{W}^{s}\left(\Phi_{1}, \Psi_{1}\right)=\mathcal{C}_{1}^{+}=\mathcal{C}_{2}^{+}$and $\mathcal{W}^{s}\left(\Psi_{1}, \Phi_{1}\right)=\mathcal{C}_{1}^{-}=\mathcal{C}_{2}^{-}$.

Proof The map $T$ is cooperative and all conditions of Theorems 1 and 4 in [19] are satisfied, which yields the existence of the global stable manifolds $\mathcal{W}^{s}\left(\Phi_{1}, \Psi_{1}\right), \mathcal{W}^{s}\left(\Psi_{1}, \Phi_{1}\right)$ and the global unstable manifolds $\mathcal{W}^{u}\left(\Phi_{1}, \Psi_{1}\right), \mathcal{W}^{u}\left(\Psi_{1}, \Phi_{1}\right)$ where $\mathcal{W}^{s}\left(\Phi_{1}, \Psi_{1}\right), \mathcal{W}^{s}\left(\Psi_{1}, \Phi_{1}\right)$ are passing through the point $E_{\mathrm{SW}}$, and they are graphs of decreasing functions. Let

$$
\begin{aligned}
\mathcal{W}^{-}= & \left\{\left(x_{0}, y_{0}\right) \mid\left(x_{0}, y_{0}\right) \preceq_{\text {ne }}\left(x_{E_{0}}, y_{E_{0}}\right) \text { for some }\left(x_{E_{0}}, y_{E_{0}}\right) \in \mathcal{W}^{s}\left(\Phi_{1}, \Psi_{1}\right) \cup \mathcal{W}^{s}\left(\Psi_{1}, \Phi_{1}\right)\right\}, \\
\mathcal{W}^{+}= & \left\{\left(x_{0}, y_{0}\right) \mid\left(x_{E_{\mathrm{NE}}}, y_{E_{\mathrm{NE}}}\right) \preceq_{\mathrm{ne}}\left(x_{0}, y_{0}\right)\right. \text { for some } \\
& \left.\left(x_{E_{\mathrm{NE}}}, y_{E_{\mathrm{NE}}}\right) \in \mathcal{W}^{s}\left(\Phi_{1}, \Psi_{1}\right) \cup \mathcal{W}^{s}\left(\Psi_{1}, \Phi_{1}\right)\right\} .
\end{aligned}
$$

Take $\left(x_{0}, y_{0}\right) \in \mathcal{W}^{-} \cap[0, \infty)^{2}$ and $\left(\tilde{x}_{0}, \tilde{y}_{0}\right) \in \mathcal{W}^{+} \cap[0, \infty)^{2}$. By Theorem 4 in [19] we see that there exist $n_{0}, n_{1}>0$ such that $T^{n}\left(x_{0}, y_{0}\right) \in \operatorname{int}\left(Q_{3}\left(\Phi_{1}, \Psi_{1}\right) \cap Q_{3}\left(\Psi_{1}, \Phi_{1}\right)\right)$ for $n>n_{0}$ and $T^{n}\left(\tilde{x}_{0}, \tilde{y}_{0}\right) \in \operatorname{int}\left(Q_{1}\left(\Phi_{1}, \Psi_{1}\right) \cap Q_{1}\left(\Psi_{1}, \Phi_{1}\right)\right)$ for $n>n_{1}$. Since $\operatorname{int}\left(Q_{3}\left(\Phi_{1}, \Psi_{1}\right) \cap Q_{3}\left(\Psi_{1}, \Phi_{1}\right)\right) \subseteq$ $\operatorname{int}\left(Q_{3}\left(E_{\mathrm{SW}}\right)\right) \subseteq \mathcal{B}\left(E_{0}\right)$ and $\operatorname{int}\left(Q_{1}\left(\Phi_{1}, \Psi_{1}\right) \cap Q_{1}\left(\Psi_{1}, \Phi_{1}\right)\right) \subseteq \operatorname{int}\left(Q_{1}\left(E_{\mathrm{SW}}\right)\right) \subseteq \mathcal{B}\left(E_{\mathrm{NE}}\right)$ we have $T^{n}\left(x_{0}, y_{0}\right) \rightarrow E_{0}$ as $n \rightarrow \infty$ if $\left(x_{0}, y_{0}\right) \in \operatorname{int}\left(Q_{3}\left(\Phi_{1}, \Psi_{1}\right) \cap Q_{3}\left(\Psi_{1}, \Phi_{1}\right)\right)$ and $T^{n}\left(x_{0}, y_{0}\right) \rightarrow E_{\mathrm{NE}}$ as $n \rightarrow \infty$ if $\left(x_{0}, y_{0}\right) \in \operatorname{int}\left(Q_{1}\left(\Phi_{1}, \Psi_{1}\right) \cap Q_{1}\left(\Psi_{1}, \Phi_{1}\right)\right)$.

Now, we consider the dynamical scenario when there exist three minimal period-two solutions. 
Theorem 8 Assume that equation (1) has three equilibrium points $U_{1} \leq \bar{x}_{0}<\bar{x}_{\mathrm{SW}}<\bar{x}_{\mathrm{NE}}$ where the equilibrium points $\bar{x}_{0}$ and $\bar{x}_{\mathrm{NE}}$ are locally asymptotically stable and $\bar{x}_{\mathrm{SW}}$ is a repeller. Further, assume that there exist three minimal period-two solutions $\left\{\Phi_{i}, \Psi_{i}\right\}$, such that $\left(\Phi_{i}, \Psi_{i}\right) \in \operatorname{int}\left(Q_{2}\left(E_{S W}\right)\right), i=1,2,3$, where $\left\{\Phi_{1}, \Psi_{1}\right\}$ and $\left\{\Phi_{2}, \Psi_{2}\right\}$ are the saddle points and $\left\{\Phi_{3}, \Psi_{3}\right\}$ is locally asymptotically stable. In this case there exist four continuous curves $\mathcal{W}^{s}\left(\Phi_{1}, \Psi_{1}\right), \mathcal{W}^{s}\left(\Psi_{1}, \Phi_{1}\right), \mathcal{W}^{s}\left(\Phi_{2}, \Psi_{2}\right), \mathcal{W}^{s}\left(\Psi_{2}, \Phi_{2}\right)$ where $\mathcal{W}^{s}\left(\Phi_{1}, \Psi_{1}\right), \mathcal{W}^{s}\left(\Psi_{1}, \Phi_{1}\right)$, $\mathcal{W}^{s}\left(\Phi_{2}, \Psi_{2}\right), \mathcal{W}^{s}\left(\Psi_{2}, \Phi_{2}\right)$ are passing through the point $E_{\mathrm{SW}}$ and are graphs of decreasing functions. Every solution which starts below $\mathcal{W}^{s}\left(\Phi_{2}, \Psi_{2}\right) \cup \mathcal{W}^{s}\left(\Psi_{2}, \Phi_{2}\right)$ in the North-East ordering converges to $E_{0}$ and every solution which starts above $W^{s}\left(\Phi_{1}, \Psi_{1}\right) \cup W^{s}\left(\Psi_{1}, \Phi_{1}\right)$ in the North-East ordering converges to $E_{\mathrm{NE}}$. Every solution which starts above $\mathcal{W}^{s}\left(\Phi_{2}, \Psi_{2}\right) \cup$ $\mathcal{W}^{s}\left(\Psi_{2}, \Phi_{2}\right)$ and below $\mathcal{W}^{s}\left(\Phi_{1}, \Psi_{1}\right) \cup \mathcal{W}^{s}\left(\Psi_{1}, \Phi_{1}\right)$ in the North-East ordering converges to $\left\{\Phi_{3}, \Psi_{3}\right\}$. In other words, the set of the initial condition $Q_{1}=\left\{\left(x_{-1}, x_{0}\right)\right\}$ is the union of five disjoint basins of attraction, namely

$$
Q_{1}=\mathcal{B}\left(E_{0}\right) \cup \mathcal{B}\left(\left\{\Phi_{1}, \Psi_{1}\right\}\right) \cup \mathcal{B}\left(\left\{\Phi_{2}, \Psi_{2}\right\}\right) \cup \mathcal{B}\left(\left\{\Phi_{3}, \Psi_{3}\right\}\right) \cup \mathcal{B}\left(E_{\mathrm{NE}}\right),
$$

where

$$
\begin{aligned}
& \mathcal{B}\left(\left\{\Phi_{1}, \Psi_{1}\right\}\right)=\mathcal{W}^{s}\left(\Phi_{1}, \Psi_{1}\right) \cup \mathcal{W}^{s}\left(\Psi_{1}, \Phi_{1}\right), \\
& \mathcal{B}\left(\left\{\Phi_{2}, \Psi_{2}\right\}\right)=\mathcal{W}^{s}\left(\Phi_{2}, \Psi_{2}\right) \cup \mathcal{W}^{s}\left(\Psi_{2}, \Phi_{2}\right), \\
& \mathcal{B}\left(E_{0}\right)=\left\{\left(x_{0}, y_{0}\right) \mid\left(x_{0}, y_{0}\right) \preceq_{\mathrm{ne}}\left(x_{E_{0}}, y_{E_{0}}\right)\right. \text { for some } \\
&\left.\left(x_{E_{0}}, y_{E_{0}}\right) \in \mathcal{W}^{s}\left(\Phi_{2}, \Psi_{2}\right) \cup \mathcal{W}^{s}\left(\Psi_{2}, \Phi_{2}\right)\right\}, \\
& \mathcal{B}\left(E_{\mathrm{NE}}\right)=\left\{\left(x_{0}, y_{0}\right) \mid\left(x_{E_{\mathrm{NE}}}, y_{E_{\mathrm{NE}}}\right) \preceq_{\mathrm{ne}}\left(x_{0}, y_{0}\right)\right. \text { for some } \\
&\left.\left(x_{E_{\mathrm{NE}}}, y_{E_{\mathrm{NE}}}\right) \in \mathcal{W}^{s}\left(\Phi_{1}, \Psi_{1}\right) \cup W^{s}\left(\Psi_{1}, \Phi_{1}\right)\right\}, \\
& \mathcal{B}\left(\left\{\Phi_{3}, \Psi_{3}\right\}\right)=\left\{\left(x_{0}, y_{0}\right) \mid\left(x_{E_{0}}, y_{E_{0}}\right) \preceq{ }_{\mathrm{ne}}\left(x_{0}, y_{0}\right) \preceq\left(x_{E_{\mathrm{NE}}}, y_{E_{\mathrm{NE}}}\right)\right. \text { for some } \\
&\left(x_{E_{\mathrm{NE}}}, y_{E_{\mathrm{NE}}}\right) \in \mathcal{W}^{s}\left(\Phi_{1}, \Psi_{1}\right) \cup W^{s}\left(\Psi_{1}, \Phi_{1}\right) \text { and } \\
&\left.\left(x_{E_{0}}, y_{E_{0}}\right) \in \mathcal{W}^{s}\left(\Phi_{2}, \Psi_{2}\right) \cup \mathcal{W}^{s}\left(\Psi_{2}, \Phi_{2}\right)\right\} .
\end{aligned}
$$

Thus, we have $\mathcal{W}^{s}\left(\Phi_{2}, \Psi_{2}\right)=\mathcal{C}_{1}^{+}, \mathcal{W}^{s}\left(\Psi_{2}, \Phi_{2}\right)=\mathcal{C}_{1}^{-}, \mathcal{W}^{s}\left(\Phi_{1}, \Psi_{1}\right)=\mathcal{C}_{2}^{+}$, and $\mathcal{W}^{s}\left(\Psi_{1}, \Phi_{1}\right)=\mathcal{C}_{2}^{-}$.

Proof All conditions of Theorems 1 and 4 in [19] for the cooperative map $T$ are satisfied, which yields the existence of the global stable manifolds $\mathcal{W}^{s}\left(\Phi_{1}, \Psi_{1}\right), \mathcal{W}^{s}\left(\Psi_{1}, \Phi_{1}\right)$, $\mathcal{W}^{s}\left(\Phi_{2}, \Psi_{2}\right), \mathcal{W}^{s}\left(\Psi_{2}, \Phi_{2}\right)$, which are passing through the point $E_{S W}$, and they are graphs of decreasing functions. Since $T$ is a cooperative map it can be seen that $\left(\Phi_{1}, \Psi_{1}\right) \ll_{\text {ne }}$ $\left(\Phi_{3}, \Psi_{3}\right) \ll_{\text {ne }}\left(\Phi_{2}, \Psi_{2}\right)$ or $\left(\Phi_{2}, \Psi_{2}\right) \ll_{\text {ne }}\left(\Phi_{3}, \Psi_{3}\right) \ll_{\text {ne }}\left(\Phi_{1}, \Psi_{1}\right)$. Assume $\left(\Phi_{1}, \Psi_{1}\right) \ll_{\text {ne }}\left(\Phi_{3}\right.$, $\left.\Psi_{3}\right) \ll_{\text {ne }}\left(\Phi_{2}, \Psi_{2}\right)$. As in the proof of Theorem 7 one can see that

$$
\begin{aligned}
\mathcal{B}\left(E_{0}\right)= & \left\{\left(x_{0}, y_{0}\right) \mid\left(x_{0}, y_{0}\right) \preceq_{\text {ne }}\left(x_{E_{0}}, y_{E_{0}}\right)\right. \text { for some } \\
& \left.\left(x_{E_{0}}, y_{E_{0}}\right) \in \mathcal{W}^{s}\left(\Phi_{1}, \Psi_{1}\right) \cup \mathcal{W}^{s}\left(\Psi_{1}, \Phi_{1}\right)\right\}, \\
\mathcal{B}\left(E_{\mathrm{NE}}\right)= & \left\{\left(x_{0}, y_{0}\right) \mid\left(x_{E_{\mathrm{NE}}}, y_{E_{\mathrm{NE}}}\right) \preceq_{\mathrm{ne}}\left(x_{0}, y_{0}\right)\right. \text { for some } \\
& \left.\left(x_{E_{\mathrm{NE}}}, y_{E_{\mathrm{NE}}}\right) \in \mathcal{W}^{s}\left(\Phi_{2}, \Psi_{2}\right) \cup W^{s}\left(\Psi_{2}, \Phi_{2}\right)\right\} .
\end{aligned}
$$


So, we assume that $\left(x_{E_{0}}, y_{E_{0}}\right) \preceq_{\text {ne }}\left(x_{0}, y_{0}\right) \preceq\left(x_{E_{\mathrm{NE}}}, y_{E_{\mathrm{NE}}}\right)$ for some $\left(x_{E_{\mathrm{NE}}}, y_{E_{\mathrm{NE}}}\right) \in \mathcal{W}^{s}\left(P_{2}\right)$ and $\left(x_{E_{0}}, y_{E_{0}}\right) \in \mathcal{W}^{s}\left(\Phi_{1}, \Psi_{1}\right)$. By Theorem 4 in [19] we see that there exists $n_{0}>0$ such that $T^{2 n}\left(x_{0}, y_{0}\right) \in \operatorname{int}\left(Q_{3}\left(\Phi_{1}, \Psi_{1}\right) \cap Q_{1}\left(\Phi_{2}, \Psi_{2}\right)\right)$ for $n>n_{0}$. By Corollary 1 we get $\llbracket\left(\Phi_{2}, \Psi_{2}\right),\left(\Phi_{3}, \Psi_{3}\right) \rrbracket \cup \llbracket\left(\Phi_{3}, \Psi_{3}\right),\left(\Phi_{1}, \Psi_{1}\right) \rrbracket \subseteq \mathcal{B}\left(\Phi_{3}, \Psi_{3}\right)$ which implies that $\operatorname{int}\left(Q_{3}\left(\Phi_{1}, \Psi_{1}\right) \cap\right.$ $\left.Q_{1}\left(\Phi_{2}, \Psi_{2}\right)\right)=\llbracket\left(\Phi_{2}, \Psi_{2}\right),\left(\Phi_{1}, \Psi_{1}\right) \rrbracket \subseteq \mathcal{B}\left(\Phi_{3}, \Psi_{3}\right)$. If $\left(x_{E_{0}}, y_{E_{0}}\right) \preceq_{\text {ne }}\left(x_{0}, y_{0}\right) \preceq\left(x_{E_{\mathrm{NE}}}, y_{E_{\mathrm{NE}}}\right)$ for some $\left(x_{E_{\mathrm{NE}}}, y_{E_{\mathrm{NE}}}\right) \in \mathcal{W}^{s}\left(\Phi_{2}, \Psi_{2}\right)$ and $\left(x_{E_{0}}, y_{E_{0}}\right) \in \mathcal{W}^{s}\left(\Phi_{1}, \Psi_{1}\right)$ then there exists $n_{0}>0$ such that $T^{2 n}\left(x_{0}, y_{0}\right) \in \operatorname{int}\left(Q_{3}\left(\Phi_{1}, \Psi_{1}\right) \cap Q_{1}\left(\Phi_{2}, \Psi_{2}\right)\right)$ for $n>n_{0}$. By Corollary 1 we get $\llbracket\left(\Psi_{2}, \Phi_{2}\right),\left(\Psi_{3}, \Phi_{3}\right) \rrbracket \cup \llbracket\left(\Psi_{3}, \Phi_{3}\right),\left(\Psi_{1}, \Phi_{1}\right) \rrbracket \subseteq \mathcal{B}\left(\Phi_{3}, \Psi_{3}\right)$, which implies that $\operatorname{int}\left(Q_{3}\left(\Psi_{1}, \Phi_{1}\right) \cap\right.$ $\left.Q_{1}\left(\Psi_{2}, \Phi_{2}\right)\right)=\llbracket\left(\Psi_{2}, \Phi_{2}\right),\left(\Psi_{1}, \Phi_{1}\right) \rrbracket \subseteq \mathcal{B}\left(\Phi_{3}, \Psi_{3}\right)$. This completes the proof.

Now, we consider two dynamical scenarios when there exists a minimal period-two solution $\{\Phi, \Psi\}$ which is a non-hyperbolic of stable type (i.e. if $\mu_{1}$ and $\mu_{2}$ are eigenvalues of $J_{T}(\Phi, \Psi)$ then $\mu_{1}=1$ and $\left.\left|\mu_{2}\right|<1\right)$.

Theorem 9 Assume that equation (1) has three equilibrium points $U_{1} \leq \bar{x}_{0}<\bar{x}_{\mathrm{SW}}<\bar{x}_{\mathrm{NE}}$ where the equilibrium points $\bar{x}_{0}$ and $\bar{x}_{\mathrm{NE}}$ are locally asymptotically stable and $\bar{x}_{\mathrm{SW}}$ is a repeller or non-hyperbolic equilibrium point. Further, assume that there exist two minimal period-two solutions $\{\Phi, \Psi\}$ and $\left\{\Phi_{1}, \Psi_{1}\right\}$, where $\{\Phi, \Psi\}$ is a non-hyperbolic period-two solution of the stable type and $\left\{\Phi_{1}, \Psi_{1}\right\}$ is a saddle point, and $(\Phi, \Psi) \ll_{\mathrm{ne}}\left(\Phi_{1}, \Psi_{1}\right)$ (see Figure 4 (a)). In this case there exist four continuous curves $\mathcal{W}^{s}\left(\Phi_{1}, \Psi_{1}\right), \mathcal{W}^{s}\left(\Psi_{1}, \Phi_{1}\right), \mathcal{C}^{s}(\Phi, \Psi)$, $\mathcal{C}^{s}(\Psi, \Phi)$ which are passing through the point $E_{S W}$ and which are graphs of decreasing functions. The set $Q_{1}=\left\{\left(x_{-1}, x_{0}\right): x_{-1} \geq U_{1}, x_{0} \geq U_{1}\right\}$ is the union of four disjoint basins of attraction, namely

$$
Q_{1}=\mathcal{B}\left(E_{0}\right) \cup \mathcal{B}\left(\left\{\Phi_{1}, \Psi_{1}\right\}\right) \cup \mathcal{B}(\{\Phi, \Psi\}) \cup \mathcal{B}\left(E_{\mathrm{NE}}\right),
$$

where

$$
\begin{aligned}
& \mathcal{B}\left(\left\{\Phi_{1}, \Psi_{1}\right\}\right)=\mathcal{W}^{s}\left(\Phi_{1}, \Psi_{1}\right) \cup \mathcal{W}^{s}\left(\Phi_{1}, \Psi_{1}\right), \\
& \mathcal{B}\left(E_{0}\right)=\left\{\left(x_{0}, y_{0}\right) \mid\left(x_{0}, y_{0}\right) \preceq \text { ne }\left(x_{E_{0}}, y_{E_{0}}\right) \text { for some }\left(x_{E_{0}}, y_{E_{0}}\right) \in \mathcal{C}(\Phi, \Psi) \cup \mathcal{C}(\Psi, \Phi)\right\},
\end{aligned}
$$

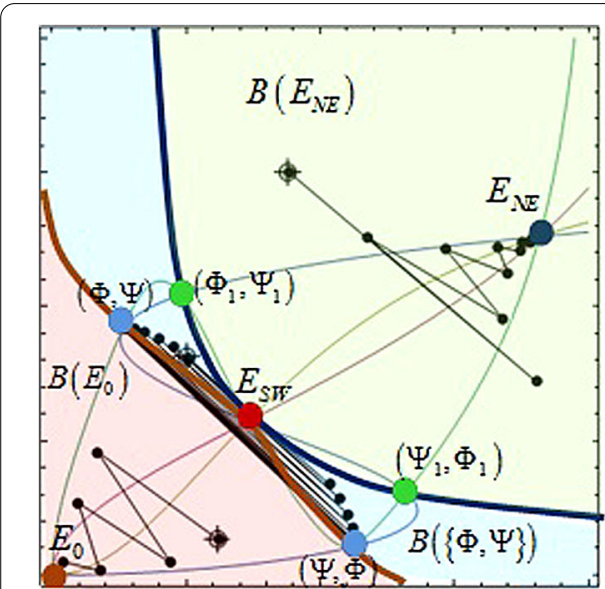

a)

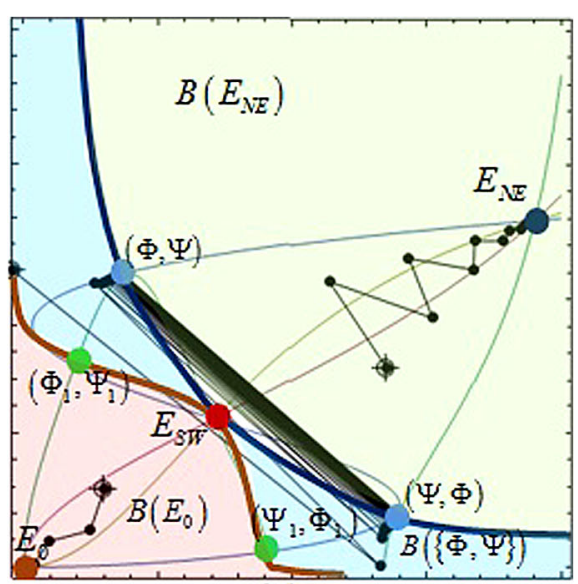

b)

Figure 4 Visual illustration of (a) Theorem 9 and (b) Theorem 10. 


$$
\begin{aligned}
& \mathcal{B}\left(E_{\mathrm{NE}}\right)=\left\{\left(x_{0}, y_{0}\right) \mid\left(x_{E_{\mathrm{NE}}}, y_{E_{\mathrm{NE}}}\right) \preceq_{\mathrm{ne}}\left(x_{0}, y_{0}\right)\right. \text { for some } \\
& \left.\left(x_{E_{\mathrm{NE}}}, y_{E_{\mathrm{NE}}}\right) \in \mathcal{W}^{s}\left(\Phi_{1}, \Psi_{1}\right) \cup W^{s}\left(\Psi_{1}, \Phi_{1}\right)\right\}, \\
& \mathcal{B}(\{\Phi, \Psi\})=\mathcal{C}(\Phi, \Psi) \cup \mathcal{C}(\Psi, \Phi) \\
& \cup\left\{\left(x_{0}, y_{0}\right) \mid\left(x_{E_{0}}, y_{E_{0}}\right) \preceq_{\text {ne }}\left(x_{0}, y_{0}\right) \preceq\left(x_{E_{\mathrm{NE}}}, y_{E_{\mathrm{NE}}}\right)\right. \text { for some } \\
& \left(x_{E_{\mathrm{NE}}}, y_{E_{\mathrm{NE}}}\right) \in \mathcal{W}^{s}\left(\Phi_{1}, \Psi_{1}\right) \cup W^{s}\left(\Psi_{1}, \Phi_{1}\right) \text { and } \\
& \left.\left(x_{E_{0}}, y_{E_{0}}\right) \in \mathcal{C}(\Phi, \Psi) \cup \mathcal{C}(\Psi, \Phi)\right\} \text {. }
\end{aligned}
$$

Thus, we have $\mathcal{C}(\Phi, \Psi)=\mathcal{C}_{1}^{+}, \mathcal{C}(\Psi, \Phi)=\mathcal{C}_{1}^{-}, \mathcal{W}^{s}\left(\Phi_{1}, \Psi_{1}\right)=\mathcal{C}_{2}^{+}$, and $\mathcal{W}^{s}\left(\Psi_{1}, \Phi_{1}\right)=\mathcal{C}_{2}^{-}$.

Proof Since $\{\Phi, \Psi\}$ is a non-hyperbolic period-two solution of the stable type and $\left\{\Phi_{1}, \Psi_{1}\right\}$ is a saddle point, all conditions of Theorems 1 and 4 in [19] for the cooperative map $T$ are satisfied, which yields the existence of the global stable manifolds $\mathcal{W}^{s}\left(\Phi_{1}, \Psi_{1}\right), \mathcal{W}^{s}\left(\Psi_{1}, \Phi_{1}\right)$ and invariant curves $\mathcal{C}(\Phi, \Psi), \mathcal{C}(\Psi, \Phi)$ which are passing through the point $E_{\mathrm{SW}}$, and they are graphs of decreasing functions.

Take $\left(x_{0}, y_{0}\right)$ such that $\left(x_{0}, y_{0}\right) \preceq_{\text {ne }}\left(x_{E_{0}}, y_{E_{0}}\right)$ for some $\left(x_{E_{0}}, y_{E_{0}}\right) \in \mathcal{C}(\Phi, \Psi) \cup \mathcal{C}(\Psi, \Phi)$. In view of Theorem 4 in [19] there exists $n_{0}>0$ such that $T^{n}\left(x_{0}, y_{0}\right) \in \operatorname{int}\left(Q_{3}(\Phi, \Psi) \cap\right.$ $\left.Q_{3}(\Psi, \Phi)\right)$ for $n>n_{0}$. Since $\operatorname{int}\left(Q_{3}(\Phi, \Psi) \cap Q_{3}(\Psi, \Phi)\right) \subseteq \operatorname{int}\left(Q_{3}\left(E_{S W}\right)\right)$ by Lemma 1 we obtain

$$
\mathcal{B}\left(E_{0}\right)=\left\{(x, y) \mid(x, y) \preceq_{\text {ne }}\left(x_{E_{0}}, y_{E_{0}}\right) \text { for some }\left(x_{E_{0}}, y_{E_{0}}\right) \in \mathcal{C}(\Phi, \Psi) \cup \mathcal{C}(\Psi, \Phi)\right\}
$$

If $\left(x_{0}, y_{0}\right)$ is such that $\left(x_{E_{\mathrm{NE}}}, y_{E_{\mathrm{NE}}}\right) \preceq_{\mathrm{ne}}\left(x_{0}, y_{0}\right)$ for some $\left(x_{E_{\mathrm{NE}}}, y_{E_{\mathrm{NE}}}\right) \in \mathcal{W}^{s}\left(\Phi_{1}, \Psi_{1}\right) \cup$ $W^{s}\left(\Psi_{1}, \Phi_{1}\right)$ by Theorem 4 in [19] there exists $n_{0}>0$ such that $T^{n}\left(x_{0}, y_{0}\right) \in \operatorname{int}\left(Q_{1}\left(\Phi_{1}, \Psi_{1}\right) \cap\right.$ $\left.Q_{1}\left(\Psi_{1}, \Phi_{1}\right)\right)$ for $n>n_{0}$. Since $\operatorname{int}\left(Q_{1}\left(\Phi_{1}, \Psi_{1}\right) \cap Q_{1}\left(\Psi_{1}, \Phi_{1}\right)\right) \subseteq \operatorname{int}\left(Q_{1}\left(E_{S W}\right)\right)$ by Lemma 1 we obtain

$$
\begin{aligned}
\mathcal{B}\left(E_{\mathrm{NE}}\right)= & \left\{\left(x_{0}, y_{0}\right) \mid\left(x_{E_{\mathrm{NE}}}, y_{E_{\mathrm{NE}}}\right) \preceq_{\mathrm{ne}}\left(x_{0}, y_{0}\right)\right. \text { for some } \\
& \left.\left(x_{E_{\mathrm{NE}}}, y_{E_{\mathrm{NE}}}\right) \in \mathcal{W}^{s}\left(\Phi_{1}, \Psi_{1}\right) \cup \mathcal{W}^{s}\left(\Psi_{1}, \Phi_{1}\right)\right\} .
\end{aligned}
$$

Now, we assume that $\left(x_{E_{0}}, y_{E_{0}}\right) \preceq_{\mathrm{ne}}\left(x_{0}, y_{0}\right) \preceq_{\mathrm{ne}}\left(x_{E_{\mathrm{NE}}}, y_{E_{\mathrm{NE}}}\right)$ for some $\left(x_{E_{\mathrm{NE}}}, y_{E_{\mathrm{NE}}}\right) \in$ $\mathcal{W}^{s}\left(\Phi_{1}, \Psi_{1}\right)$ and $\left(x_{E_{0}}, y_{E_{0}}\right) \in \mathcal{C}(\Phi, \Psi)$. By Theorem 4 in [19] we see that there exists $n_{0}>0$ such that $T^{n}\left(x_{0}, y_{0}\right) \in \operatorname{int}\left(Q_{3}\left(\Phi_{1}, \Psi_{1}\right) \cap Q_{1}(\Phi, \Psi)\right)$ for $n>n_{0}$. By Corollary 1 we get $\llbracket(\Phi, \Psi),\left(\Phi_{1}, \Psi_{1}\right) \rrbracket \subseteq \mathcal{B}(\{\Phi, \Psi\})$. Similarly, if $\left(x_{E_{0}}, y_{E_{0}}\right) \preceq_{\text {ne }}\left(x_{0}, y_{0}\right) \preceq_{\text {ne }}\left(x_{E_{\mathrm{NE}}}, y_{E_{\mathrm{NE}}}\right)$ for some $\left(x_{E_{\mathrm{NE}}}, y_{E_{\mathrm{NE}}}\right) \in \mathcal{W}^{s}\left(\Psi_{1}, \Phi_{1}\right)$ and $\left(x_{E_{0}}, y_{E_{0}}\right) \in \mathcal{C}(\Psi, \Phi)$ we see that there exists $n_{0}>0$ such that $T^{n}\left(x_{0}, y_{0}\right) \in \operatorname{int}\left(Q_{3}\left(\Psi_{1}, \Phi_{1}\right) \cap Q_{1}(\Psi, \Phi)\right)$ for $n>n_{0}$. By Corollary 1 we get $\llbracket T(P),\left(\Psi_{1}, \Phi_{1}\right) \rrbracket \subseteq$ $\mathcal{B}(\{\Phi, \Psi\})$. This implies $\left(x_{0}, y_{0}\right) \in \mathcal{B}(\{\Phi, \Psi\})$.

The proof of the following result is similar to the proof of Theorem 9 and will be omitted.

Theorem 10 Assume that equation (1) has three equilibrium points $U_{1} \leq \bar{x}_{0}<\bar{x}_{\mathrm{SW}}<\bar{x}_{\mathrm{NE}}$ where the equilibrium points $\bar{x}_{0}$ and $\bar{x}_{\mathrm{NE}}$ are locally asymptotically stable and $\bar{x}_{\mathrm{SW}}$ is a repeller or non-hyperbolic equilibrium point. Further, assume that there exist two minimal period-two solutions $\{\Phi, \Psi\}$ and $\left\{\Phi_{1}, \Psi_{1}\right\}$, where $\{\Phi, \Psi\}$ is a non-hyperbolic period-two solution of the stable type and $\left\{\Phi_{1}, \Psi_{1}\right\}$ is a saddle point, and $\left(\Phi_{1}, \Psi_{1}\right) \ll_{\mathrm{ne}}(\Phi, \Psi)$ (see Figure 4(b)). In this case there exist four continuous curves $\mathcal{W}^{s}\left(\Phi_{1}, \Psi_{1}\right), \mathcal{W}^{s}\left(\Psi_{1}, \Phi_{1}\right), \mathcal{C}^{s}(\Phi, \Psi)$, 
$\mathcal{C}^{s}(\Psi, \Phi)$ where $\mathcal{W}^{s}\left(\Phi_{1}, \Psi_{1}\right), \mathcal{W}^{s}\left(\Psi_{1}, \Phi_{1}\right), \mathcal{C}(\Phi, \Psi), \mathcal{C}(\Psi, \Phi)$ are passing through the point $E_{\mathrm{SW}}$, which are graphs of decreasing functions. The set $Q_{1}=\left\{\left(x_{-1}, x_{0}\right): x_{-1} \geq U_{1}, x_{0} \geq U_{1}\right\}$ is the union of four disjoint basins of attraction, namely

$$
Q_{1}=\mathcal{B}\left(E_{0}\right) \cup \mathcal{B}\left(\left\{\Phi_{1}, \Psi_{1}\right\}\right) \cup \mathcal{B}(\{\Phi, \Psi\}) \cup \mathcal{B}\left(E_{\mathrm{NE}}\right)
$$

where

$$
\begin{aligned}
& \mathcal{B}\left(\left\{\Phi_{1}, \Psi_{1}\right\}\right)= \mathcal{W}^{s}\left(\Phi_{1}, \Psi_{1}\right) \cup \mathcal{W}^{s}\left(\Phi_{1}, \Psi_{1}\right), \\
& \mathcal{B}\left(E_{0}\right)=\left\{\left(x_{0}, y_{0}\right) \mid\left(x_{0}, y_{0}\right) \preceq_{\mathrm{ne}}\left(x_{E_{0}}, y_{E_{0}}\right)\right. \text { for some } \\
&\left.\quad\left(x_{E_{0}}, y_{E_{0}}\right) \in \mathcal{W}^{s}\left(\Phi_{1}, \Psi_{1}\right) \cup W^{s}\left(\Psi_{1}, \Phi_{1}\right)\right\}, \\
& \mathcal{B}\left(E_{\mathrm{NE}}\right)=\left\{\left(x_{0}, y_{0}\right) \mid\left(x_{E_{\mathrm{NE}}}, y_{E_{\mathrm{NE}}}\right) \preceq_{\mathrm{ne}}(x, y) \text { for some }\left(x_{E_{\mathrm{NE}}}, y_{E_{\mathrm{NE}}}\right) \in \mathcal{C}(\Phi, \Psi) \cup \mathcal{C}(\Psi, \Phi)\right\}, \\
& \mathcal{B}(\{\Phi, \Psi\})= \mathcal{C}(\Phi, \Psi) \cup \mathcal{C}(\Psi, \Phi) \\
& \cup\left\{\left(x_{0}, y_{0}\right) \mid\left(x_{E_{0}}, y_{E_{0}}\right) \preceq_{\mathrm{ne}}\left(x_{0}, y_{0}\right) \preceq\left(x_{E_{\mathrm{NE}}}, y_{E_{\mathrm{NE}}}\right)\right. \text { for some } \\
&\left(x_{E_{\mathrm{NE}}}, y_{E_{\mathrm{NE}}}\right) \in \mathcal{C}(\Phi, \Psi) \cup \mathcal{C}(\Psi, \Phi) \text { and } \\
&\left.\left(x_{E_{0}}, y_{E_{0}}\right) \in \mathcal{W}^{s}\left(\Phi_{1}, \Psi_{1}\right) \cup W^{s}\left(\Psi_{1}, \Phi_{1}\right)\right\} .
\end{aligned}
$$

Thus, we have $\mathcal{C}(\Phi, \Psi)=\mathcal{C}_{2}^{+}, \mathcal{C}(\Psi, \Phi)=\mathcal{C}_{2}^{-}, \mathcal{W}^{s}\left(\Phi_{1}, \Psi_{1}\right)=\mathcal{C}_{1}^{+}$, and $\mathcal{W}^{s}\left(\Psi_{1}, \Phi_{1}\right)=\mathcal{C}_{1}^{-}$.

\section{Examples}

In this section we give an application of our results in establishing global dynamics of some equations from mathematical biology. We present two cases in detail. All three models are of the types

$$
x_{n+1}=f_{1}\left(x_{n}\right)+f_{2}\left(x_{n-1}\right), \quad n=0,1, \ldots
$$

where $f_{i}, i=1,2$ are transition functions, considered by many researchers in mathematical biology [6]. The most common transition functions in modeling are linear, Beverton-Holt $\left(f(u)=\frac{a u}{1+u}\right)$, and sigmoid Beverton-Holt $\left(f(u)=\frac{a u^{\sigma}}{1+u^{\sigma}}, \sigma \geq 1\right)$. The case when both transition functions are Beverton-Holt functions was treated in [6] and we prove that in this case there are no period-two solutions and so every solution converges to an equilibrium. However, in all other cases for any other combination of transition functions the periodtwo solutions exist and play an important role in the dynamics.

4.1 Example 1: $x_{n+1}=\frac{A x_{n}^{2}}{1+x_{n}^{2}}+\frac{B x_{n-1}^{2}}{1+x_{n-1}^{2}}$

In this part we apply Theorems 5-10 to describe the global dynamics of the difference equation in the title.

\subsubsection{The equilibrium points}

We consider the difference equation

$$
x_{n+1}=\frac{A x_{n}^{2}}{1+x_{n}^{2}}+\frac{B x_{n-1}^{2}}{1+x_{n-1}^{2}}, \quad n=0,1, \ldots
$$


where $A, B>0$ and the initial conditions $x_{-1}, x_{0}$ are non-negative. In view of the above restriction on the initial conditions of equation (8), the equilibrium points of equation (8) are the nonnegative solutions of the equation

$$
\bar{x}=\frac{A \bar{x}^{2}}{1+\bar{x}^{2}}+\frac{B \bar{x}^{2}}{1+\bar{x}^{2}}
$$

or equivalently

$$
\bar{x}^{3}-\bar{x}^{2}(A+B)+\bar{x}=0 .
$$

Now we have the following result.

Lemma 3 The following holds:

(i) If $A+B<2$ then equation (8) has a unique equilibrium point $x_{0}=0$.

(ii) If $A+B=2$ then equation (8) has two equilibrium points $x_{0}=0$ and $x=(A+B) / 2$.

(iii) If $A+B>2$ then equation (8) has three equilibrium points $x_{0}=0$,

$$
x_{\mathrm{SW}}=\frac{1}{2}\left(A+B-\sqrt{(A+B)^{2}-4}\right) \text {, and } x_{\mathrm{NE}}=\frac{1}{2}\left(A+B+\sqrt{(A+B)^{2}-4}\right) ; x=(A+B) / 2 \text {. }
$$

Next, we investigate the stability of the nonnegative equilibrium points of equation (8). Set

$$
f(u, v)=\frac{A u^{2}}{1+u^{2}}+\frac{B v^{2}}{1+v^{2}}
$$

and observe that $f_{u}(u, v)>0$ and $f_{v}(u, v)>0$. The next three lemmas are straightforward.

Lemma 4 Assume that $A, B>0$. Then the equilibrium point $x_{0}$ is locally asymptotically stable.

Lemma 5 Assume that $A+B \geq 2$. Then the equilibrium point $x_{\mathrm{NE}}$ is locally asymptotically stable if $A+B>2$ and non-hyperbolic if $A+B=2$.

Proof It can be seen that

$$
p=f_{u}\left(x_{\mathrm{NE}}, x_{\mathrm{NE}}\right)=\frac{4 A}{(A+B)^{2}\left(\sqrt{(A+B)^{2}-4}+A+B\right)}
$$

and

$$
q=f_{v}\left(x_{\mathrm{NE}}, x_{\mathrm{NE}}\right)=\frac{4 B}{(A+B)^{2}\left(\sqrt{(A+B)^{2}-4}+A+B\right)} .
$$

Then the proof follows from Theorem 1.1.1 in [2] and the fact that

$$
1-p-q=\frac{\sqrt{(A+B)^{2}-4}}{A+B} \text { and } p, q>0 .
$$

Lemma 6 Assume that $A+B \geq 2$. Then the equilibrium point $x_{\mathrm{SW}}$ is:

(i) a saddle point if $2 A(A+B)+(A-B) \sqrt{(A+B)^{2}-4}>0$ and $A+B>2$;

(ii) a repeller if $2 A(A+B)+(A-B) \sqrt{(A+B)^{2}-4}<0$ and $A+B>2$;

(iii) a non-hyperbolic if $A+B=2$ or $2 A(A+B)+(A-B) \sqrt{(A+B)^{2}-4}=0$. 
Proof It can be seen that

$$
\begin{aligned}
& p=f_{u}\left(x_{\mathrm{SW}}, x_{\mathrm{SW}}\right)=\frac{A\left(\sqrt{(A+B)^{2}-4}+A+B\right)}{(A+B)^{2}}, \\
& q=f_{v}\left(x_{\mathrm{SW}}, x_{\mathrm{SW}}\right)=\frac{B\left(\sqrt{(A+B)^{2}-4}+A+B\right)}{(A+B)^{2}} .
\end{aligned}
$$

Then the proof follows from Theorem 1.1.1 in [2] and the fact that

$$
1-p-q=-\frac{\sqrt{(A+B)^{2}-4}}{A+B} \text { and } 1+p-q=\frac{2 A(A+B)+(A-B) \sqrt{(A+B)^{2}-4}}{(A+B)^{2}} .
$$

\subsubsection{Period-two solutions}

Next, we investigate the existence and stability of the positive minimal period-two solutions of equation (8). Let $\{\phi, \psi\}$ be a minimal period-two solution of equation (8). Then

$$
\phi=f(\psi, \phi) \text { and } \psi=f(\phi, \psi) \text { with } \psi, \phi \in[0, \infty) \text { and } \phi \neq \psi \text {, }
$$

which is equivalent to

$$
\phi=\frac{A \psi^{2}}{1+\psi^{2}}+\frac{B \phi^{2}}{1+\phi^{2}} \quad \text { and } \quad \psi=\frac{A \phi^{2}}{1+\phi^{2}}+\frac{B \psi^{2}}{1+\psi^{2}} \quad \text { with } \phi \neq \psi
$$

which is true if and only if $\phi \neq \psi$,

$$
-A \psi^{2}-A \psi^{2} \phi^{2}-B \psi^{2} \phi^{2}-B \phi^{2}+\psi^{2} \phi^{3}+\phi^{3}+\psi^{2} \phi+\phi=0
$$

and

$$
\psi-A \psi^{2} \phi^{2}-A \phi^{2}-B \psi^{2}-B \psi^{2} \phi^{2}+\psi^{3}+\psi+\psi^{3} \phi^{2}+\psi \phi^{2}=0
$$

By eliminating $\psi$ from (10) and (11) we obtain

$$
\phi\left(\phi^{2}+1\right)\left(A \phi+B \phi-\phi^{2}-1\right) \tilde{f}(\phi)=0
$$

and by eliminating $\phi$ from (10) and (11) we obtain

$$
\psi\left(\psi^{2}+1\right)\left(A \psi+B \psi-\psi^{2}-1\right) \tilde{f}(\psi)=0,
$$

where

$$
\begin{aligned}
\tilde{f}(x)= & B^{2} x^{6}+2 B x^{5}\left(A^{2}-B^{2}\right)+x^{4}\left(A^{4}-2 A^{2} B^{2}+A^{2}+B^{4}+2 B^{2}\right) \\
& +x^{3}\left(A^{3}+2 A^{2} B-A B^{2}-2 B^{3}\right)+x^{2}\left(A^{4}-A^{3} B-A^{2}\left(B^{2}-2\right)+A B^{3}+B^{2}\right) \\
& +x\left(A^{3}-A B^{2}\right)+A^{2} .
\end{aligned}
$$

Since $(A+B) x-x^{2}-1 \neq 0$ for any $x$ different from the equilibrium point, the minimal period-two solutions are solutions of the equation

$$
\tilde{f}(x)=0 .
$$




\section{Lemma 7 Let}

$$
\begin{aligned}
\Delta= & 4 A^{6}-12 A^{5} B+8 A^{4} B^{2}+8 A^{4}+8 A^{3} B^{3}-36 A^{3} B-12 A^{2} B^{4}+47 A^{2} B^{2}+4 A^{2} \\
& +4 A B^{5}-18 A B^{3}+8 A B-B^{4}+4 B^{2}, \\
\Delta_{1}= & 2 A^{4}-4 A^{2} B^{2}-3 A^{2}+2 B^{4}-6 B^{2}, \\
\Delta_{2}= & -\left(8 A^{7}-8 A^{5} B^{2}+16 A^{5}-32 A^{4} B-8 A^{3} B^{4}-45 A^{3} B^{2}+8 A^{3}+39 A^{2} B^{3}\right) \\
& +8 A^{2} B+8 A B^{6}-40 A B^{4}+16 A B^{2}-4 B^{5}+16 B^{3}, \\
\Delta_{3}= & 32 A^{12}-64 A^{11} B-96 A^{10} B^{2}+148 A^{10}+256 A^{9} B^{3}-436 A^{9} B+64 A^{8} B^{4} \\
& +332 A^{8} B^{2}+264 A^{8}-384 A^{7} B^{5}-92 A^{7} B^{3}-900 A^{7} B \\
& +64 A^{6} B^{6}+324 A^{6} B^{4}+1,627 A^{6} B^{2}+212 A^{6} \\
& +256 A^{5} B^{7}-84 A^{5} B^{5}-1,992 A^{5} B^{3}-624 A^{5} B-96 A^{4} B^{8} \\
& -700 A^{4} B^{6}+1,402 A^{4} B^{4}+2,008 A^{4} B^{2} \\
& +64 A^{4}-64 A^{3} B^{9}+652 A^{3} B^{7}-192 A^{3} B^{5}-1,536 A^{3} B^{3} \\
& +32 A^{2} B^{10}-104 A^{2} B^{8}-425 A^{2} B^{6}+724 A^{2} B^{4} \\
& -128 A^{2} B^{2}-40 A B^{9}+204 A B^{7}-144 A B^{5}+12 B^{8}-64 B^{6}+64 B^{4}, \\
\Delta_{4}= & \Delta\left(4 A^{9}-16 A^{7} B^{2}+15 A^{7}-23 A^{6} B+24 A^{5} B^{4}+10 A^{5} B^{2}\right. \\
& +7 A^{5}-20 A^{4} B^{3}-77 A^{4} B-16 A^{3} B^{6}-A^{3} B^{4}+69 A^{3} B^{2} \\
& -8 A^{3}+45 A^{2} B^{5}-77 A^{2} B^{3}+8 A^{2} B+4 A B^{8} \\
& \left.-24 A B^{6}+20 A B^{4}+8 A B^{2}-2 B^{7}+10 B^{5}-8 B^{3}\right), \\
\Delta_{5}= & -3 A^{4}-8 A^{3} B-6 A^{2} B^{2}-4 A^{2}+8 A B+B^{4}-4 B^{2} . \\
&
\end{aligned}
$$

\section{Then the following holds:}

(a) Consider equation (12). Then all its real roots are positive numbers. Furthermore, equation (8) has up to three minimal period-two solutions.

(b) If $\Delta_{i}>0$, for all $1 \leq i \leq 5$ and $\Delta>0$ then equation (12) has six real roots.

Consequently, equation (8) has three minimal period-two solutions.

(c) If $\Delta_{i} \leq 0$ for some $1 \leq i \leq 4$ and $\Delta_{5}>0, \Delta>0$ then equation (12) has two distinct real roots and two pairs of conjugate imaginary roots. Consequently, equation (8) has one minimal period-two solution.

(d) If $\Delta_{i}>0$ for all $1 \leq i \leq j-1$ and $\Delta_{i}<0$ for all $j \leq i \leq 4$ for some $1 \leq j \leq 5$ and $\Delta_{5}<0$, $\Delta>0$ then equation (12) has four distinct real roots and one pair of conjugate imaginary roots. Consequently, equation (8) has two minimal period-two solutions.

(e) If $\Delta_{i} \leq 0, \Delta_{i+1} \geq 0$ for some $1 \leq i \leq 3$ and $\Delta_{5}<0, \Delta>0$ then equation (12) has three pairs of conjugate imaginary roots. Consequently, equation (8) has no minimal period-two solution.

(f) Assume that $\Delta=0$, and $\Delta_{3} \neq 0$ and $\Delta_{5} \neq 0$.

(f.1) If $\left(\Delta_{1} \leq 0\right.$ and $\left.\Delta_{2} \geq 0\right)$ or $\left(\Delta_{2} \leq 0\right.$ and $\left.\Delta_{3}>0\right)$ then equation (12) has no real roots and has two distinct pairs of conjugate imaginary roots, one of them of 
multiplicity one and the other one of multiplicity two. Consequently, equation (8) has no minimal period-two solutions.

(f.2) If $\Delta_{1}>0$ and $\Delta_{2}>0$ and $\Delta_{3}>0$ then equation (12) has four distinct real roots, two of them are multiplicity two and other two of multiplicity one and has no conjugate imaginary roots. Consequently, equation (8) has two minimal period-two solutions.

Proof The proof of (a) follows from Descartes' rule of signs.

The discrimination matrix $[22,23]$ of $\tilde{f}(x)$ and $\tilde{f}^{\prime}(x)$ is given by

$$
\operatorname{Discr}(\tilde{f})=\left(\begin{array}{cccccccccccc}
a_{6} & a_{5} & a_{4} & a_{3} & a_{2} & a_{1} & a_{0} & 0 & 0 & 0 & 0 & 0 \\
0 & 6 a_{6} & 5 a_{5} & 4 a_{4} & 3 a_{3} & 2 a_{2} & a_{1} & 0 & 0 & 0 & 0 & 0 \\
0 & a_{6} & a_{5} & a_{4} & a_{3} & a_{2} & a_{1} & a_{0} & 0 & 0 & 0 & 0 \\
0 & 0 & 6 a_{6} & 5 a_{5} & 4 a_{4} & 3 a_{3} & 2 a_{2} & a_{1} & 0 & 0 & 0 & 0 \\
0 & 0 & a_{6} & a_{5} & a_{4} & a_{3} & a_{2} & a_{1} & a_{0} & 0 & 0 & 0 \\
0 & 0 & 0 & 6 a_{6} & 5 a_{5} & 4 a_{4} & 3 a_{3} & 2 a_{2} & a_{1} & 0 & 0 & 0 \\
0 & 0 & 0 & a_{6} & a_{5} & a_{4} & a_{3} & a_{2} & a_{1} & a_{0} & 0 & 0 \\
0 & 0 & 0 & 0 & 6 a_{6} & 5 a_{5} & 4 a_{4} & 3 a_{3} & 2 a_{2} & a_{1} & 0 & 0 \\
0 & 0 & 0 & 0 & a_{6} & a_{5} & a_{4} & a_{3} & a_{2} & a_{1} & a_{0} & 0 \\
0 & 0 & 0 & 0 & 0 & 6 a_{6} & 5 a_{5} & 4 a_{4} & 3 a_{3} & 2 a_{2} & a_{1} & 0 \\
0 & 0 & 0 & 0 & 0 & a_{6} & a_{5} & a_{4} & a_{3} & a_{2} & a_{1} & a_{0} \\
0 & 0 & 0 & 0 & 0 & 0 & 6 a_{6} & 5 a_{5} & 4 a_{4} & 3 a_{3} & 2 a_{2} & a_{1}
\end{array}\right),
$$

where $a_{6}=B^{2}, a_{5}=2 B\left(A^{2}-B^{2}\right), a_{4}=A^{4}-2 A^{2} B^{2}+A^{2}+B^{4}+2 B^{2}, a_{3}=A^{3}+2 A^{2} B-A B^{2}-$ $2 B^{3}, a_{2}=A^{4}-A^{3} B-A^{2}\left(B^{2}-2\right)+A B^{3}+B^{2}, a_{1}=A^{3}-A B^{2}$, and $a_{0}=A^{2}$.

Let $D_{k}$ denote the determinant of the submatrix of $\operatorname{Discr}(\tilde{f})$, formed by the first $2 k$ row and the first $2 k$ columns, for $k=1, \ldots, m$. By a straightforward calculation one can see that

$$
\begin{aligned}
D_{1}= & 6 B^{4}, \\
D_{2}= & 4 B^{6}\left(2 A^{4}-4 A^{2} B^{2}-3 A^{2}+2 B^{4}-6 B^{2}\right), \\
D_{3}= & -2 B^{6}(A-B)^{2}(A+B)\left(8 A^{7}-8 A^{5} B^{2}+16 A^{5}-32 A^{4} B\right. \\
& -8 A^{3} B^{4}-45 A^{3} B^{2}+8 A^{3}+39 A^{2} B^{3} \\
& \left.+8 A^{2} B+8 A B^{6}-40 A B^{4}+16 A B^{2}-4 B^{5}+16 B^{3}\right), \\
D_{4}= & A^{2} B^{6}(B-A)^{2}(A+B)^{2}\left(32 A^{12}-64 A^{11} B-96 A^{10} B^{2}+148 A^{10}+256 A^{9} B^{3}\right. \\
& -436 A^{9} B+64 A^{8} B^{4}+332 A^{8} B^{2}+264 A^{8}-384 A^{7} B^{5}-92 A^{7} B^{3} \\
& -900 A^{7} B+64 A^{6} B^{6}+324 A^{6} B^{4}+1,627 A^{6} B^{2}+212 A^{6} \\
& +256 A^{5} B^{7}-84 A^{5} B^{5}-1,992 A^{5} B^{3}-624 A^{5} B-96 A^{4} B^{8}-700 A^{4} B^{6} \\
& +1,402 A^{4} B^{4}+2,008 A^{4} B^{2}+64 A^{4}-64 A^{3} B^{9}+652 A^{3} B^{7}-192 A^{3} B^{5} \\
& -1,536 A^{3} B^{3}+32 A^{2} B^{10}-104 A^{2} B^{8}-425 A^{2} B^{6}+724 A^{2} B^{4} \\
& \left.-128 A^{2} B^{2}-40 A B^{9}+204 A B^{7}-144 A B^{5}+12 B^{8}-64 B^{6}+64 B^{4}\right), \\
D_{5}= & A^{4} B^{6}(B-A)^{4}(A+B)^{3}\left(4 A^{6}-12 A^{5} B+8 A^{4} B^{2}+8 A^{4}+8 A^{3} B^{3}-36 A^{3} B\right.
\end{aligned}
$$




$$
\begin{aligned}
& \left.-12 A^{2} B^{4}+47 A^{2} B^{2}+4 A^{2}+4 A B^{5}-18 A B^{3}+8 A B-B^{4}+4 B^{2}\right) \\
& \times\left(4 A^{9}-16 A^{7} B^{2}+15 A^{7}-23 A^{6} B+24 A^{5} B^{4}+10 A^{5} B^{2}\right. \\
& +7 A^{5}-20 A^{4} B^{3}-77 A^{4} B-16 A^{3} B^{6}-A^{3} B^{4}+69 A^{3} B^{2} \\
& -8 A^{3}+45 A^{2} B^{5}-77 A^{2} B^{3}+8 A^{2} B+4 A B^{8} \\
& \left.-24 A B^{6}+20 A B^{4}+8 A B^{2}-2 B^{7}+10 B^{5}-8 B^{3}\right), \\
D_{6}= & A^{6} B^{6}(B-A)^{6}(A+B)^{4}\left(-3 A^{4}-8 A^{3} B-6 A^{2} B^{2}-4 A^{2}+8 A B+B^{4}-4 B^{2}\right) \\
& \times\left(4 A^{6}-12 A^{5} B+8 A^{4} B^{2}+8 A^{4}+8 A^{3} B^{3}-36 A^{3} B-12 A^{2} B^{4}+47 A^{2} B^{2}\right. \\
& \left.+4 A^{2}+4 A B^{5}-18 A B^{3}+8 A B-B^{4}+4 B^{2}\right)^{2} .
\end{aligned}
$$

The rest of the proof follows from Theorem 1 in [23].

\subsubsection{The global behavior}

In this section we describe the global behavior of equation (8) which has three equilibrium points $\bar{x}_{0}, \bar{x}_{\mathrm{SW}}, \bar{x}_{\mathrm{NE}} \in I$ such that $0=\bar{x}_{0}<\bar{x}_{\mathrm{SW}}<\bar{x}_{\mathrm{NE}}$ where the equilibrium points $\bar{x}_{0}$ and $\bar{x}_{\mathrm{NE}}$ are locally asymptotically stable and $\bar{x}_{\mathrm{SW}}$ is unstable. Further, $x_{n}<A+B$ for all $n \geq 1$. One can see that all minimal period-two solutions of (8) belong to $\operatorname{int}\left(Q_{2}\left(E_{\mathrm{SW}}\right) \cup Q_{4}\left(E_{\mathrm{SW}}\right)\right)$.

Lemma 8 If $A+B<2$ then there exists a unique equilibrium point $x_{0}=0$ which is globally asymptotically stable.

Proof The proof follows from Theorem 2 and the fact that $x_{n}<A+B$ for $n \geq 1$.

Theorem 11 Assume that $A+B>2$. Then the following holds:

(i) If $\Delta_{i} \leq 0$ and $\Delta_{i+1} \geq 0$ for some $1 \leq i \leq 3$ and $\Delta_{5}<0, \Delta>0$ then equation (8) has three equilibrium points such that $0=\bar{x}_{0}<\bar{x}_{\mathrm{SW}}<\bar{x}_{\mathrm{NE}}$ where $x_{0}$ and $x_{\mathrm{NE}}$ are locally asymptotically stable and $x_{\mathrm{SW}}$ is saddle point and has no period-two solution. The global behavior of equation (8) is described by Theorem 6. For example, this happens for $A=0.46$ and $B=1.98$.

(ii) If $\Delta_{i} \leq 0$ for some $1 \leq i \leq 4$ and $\Delta_{5}>0, \Delta>0$ then equation (8) has three equilibrium points such that $0=\bar{x}_{0}<\bar{x}_{\mathrm{SW}}<\bar{x}_{\mathrm{NE}}$ where $x_{0}$ and $x_{\mathrm{NE}}$ are locally asymptotically stable and $x_{\mathrm{SW}}$ is repeller and one period-two solution $\left\{\phi_{1}, \psi_{1}\right\}$ which is a saddle point. The global behavior of equation (8) is described by Theorem 7. For example, this happens for $A=0.46$ and $B=2.18$.

(iii) If $\Delta_{i}>0$, for all $1 \leq i \leq 5$ and $\Delta>0$ then equation (8) has three equilibrium points such that $0=\bar{x}_{0}<\bar{x}_{\mathrm{SW}}<\bar{x}_{\mathrm{NE}}$ where $x_{0}$ and $x_{\mathrm{NE}}$ are locally asymptotically stable and $x_{\mathrm{SW}}$ is repeller and three minimal period-two solutions $\left\{\phi_{1}, \psi_{1}\right\},\left\{\phi_{2}, \psi_{2}\right\}$, and $\left\{\phi_{3}, \psi_{3}\right\}$ such that $\left(\phi_{1}, \psi_{1}\right) \preceq_{\text {ne }}\left(\phi_{2}, \psi_{2}\right) \preceq_{\text {ne }}\left(\phi_{3}, \psi_{3}\right)$ where $\left\{\phi_{1}, \psi_{1}\right\}$, and $\left\{\phi_{3}, \psi_{3}\right\}$ are saddle points and $\left\{\phi_{2}, \psi_{2}\right\}$ is locally asymptotically stable. The global behavior of equation (8) is described by Theorem 8. For example, this happens for $A=0.06$ and $B=2.09$.

(iv) If $\Delta=0, \Delta_{5}>0, \Delta_{1}>0, \Delta_{2}>0, \Delta_{3}>0$ then equation (8) has three equilibrium points such that $0=\bar{x}_{0}<\bar{x}_{\mathrm{SW}}<\bar{x}_{\mathrm{NE}}$ where $x_{0}$ and $x_{\mathrm{NE}}$ are locally asymptotically stable and $x_{\mathrm{SW}}$ is repeller and two minimal period-two solutions $\left\{\phi_{1}, \psi_{1}\right\}$ and 
$\left\{\phi_{2}, \psi_{2}\right\}$ such that $\left(\phi_{1}, \psi_{1}\right) \preceq_{\text {ne }}\left(\phi_{2}, \psi_{2}\right)$ where either $\left\{\phi_{1}, \psi_{1}\right\}$ is non-hyperbolic and $\left\{\phi_{2}, \psi_{2}\right\}$ is a saddle point or $\left\{\phi_{1}, \psi_{1}\right\}$ is a saddle point and $\left\{\phi_{2}, \psi_{2}\right\}$ is non-hyperbolic. If period-two solution which is non-hyperbolic is of stable type then the global behavior of equation (8) is described by Theorems 8 and 9. For example, this happens for $A=0.06$ and $B=1.9998768381155188$.

Proof (i) By assumption we have $\Delta_{5}<0$. By Lemmas 4 and 5 the equilibrium points $x_{0}$ and $x_{\mathrm{NE}}$ are locally asymptotically stable. Since

$$
\begin{aligned}
& \left(2 A^{2}+2 A B\right)^{2}-(A-B)^{2}\left((A+B)^{2}-4\right) \\
& \quad=3 A^{4}+8 A^{3} B+6 A^{2} B^{2}+4 A^{2}-8 A B-B^{4}+4 B^{2}
\end{aligned}
$$

by Lemma 6 , the equilibrium point $x_{\mathrm{SW}}$ is a saddle point. By Lemma 7 there are no minimal period-two solutions so the rest of the proof follows from Theorem 6.

(ii) By our assumptions we have $\Delta_{5}>0$. By Lemmas 4 and 5 the equilibrium points $x_{0}$ and $x_{\mathrm{NE}}$ are locally asymptotically stable. In view of (13) and Lemma 6 the equilibrium point $x_{\mathrm{SW}}$ is a repeller. Since the discriminant of polynomial $\tilde{f}(x)$ is $\operatorname{Dis}(\tilde{f})=D_{6} \neq 0$ similarly as in Theorem 15 of [21] one can see that all period-two solutions are hyperbolic. In view of Lemma 2 we see that $\mathcal{C}_{1}^{+} \cup \mathcal{C}_{1}^{-}$is a totally ordered set which is invariant under $T$. If $\left(x_{0}, y_{0}\right) \in \mathcal{C}_{1}^{+} \cup \mathcal{C}_{1}^{-}$then $\left\{T^{n}\left(x_{0}, y_{0}\right)\right\}$ is eventually componentwise monotone. Then there exists a minimal period-two solution $\left\{\left(\phi_{1}, \psi_{1}\right),\left(\psi_{1}, \phi_{1}\right)\right\} \in \mathcal{C}_{1}^{+} \cup \mathcal{C}_{1}^{-} \subset Q_{2}\left(E_{S W}\right) \cup Q_{4}\left(E_{S W}\right)$ such that $T^{n}\left(x_{0}, y_{0}\right) \rightarrow\left(\phi_{1}, \psi_{1}\right)$ as $n \rightarrow \infty$. By Lemma 7 there exists only one minimal periodtwo solution, which implies that $\left\{\phi_{1}, \psi_{1}\right\}$ is a saddle point. The rest of the proof follows from Theorem 7.

(iii) Similarly as in (ii) one can see that $\left(\phi_{1}, \psi_{1}\right) \in \mathcal{C}_{1}^{+} \cup \mathcal{C}_{1}^{-}$and $\left(\phi_{3}, \psi_{3}\right) \in \mathcal{C}_{2}^{+} \cup \mathcal{C}_{2}^{-}$which are saddle points. By Corollary 1 there exists a minimal period-two solution $\left\{\phi_{2}, \psi_{2}\right\}$ such that $\left(\phi_{1}, \psi_{1}\right) \preceq_{\text {ne }}\left(\phi_{2}, \psi_{2}\right) \preceq_{\text {ne }}\left(\phi_{3}, \psi_{3}\right)$ which is locally asymptotically stable. The rest of the proof follows from Theorem 8 .

(iv) Since $\Delta_{5}>0$, by Lemmas 4 and 5 the equilibrium points $x_{0}$ and $x_{\mathrm{NE}}$ are locally asymptotically stable. Since

$$
\begin{aligned}
& \left(2 A^{2}+2 A B\right)^{2}-(A-B)^{2}\left((A+B)^{2}-4\right) \\
& \quad=3 A^{4}+8 A^{3} B+6 A^{2} B^{2}+4 A^{2}-8 A B-B^{4}+4 B^{2}
\end{aligned}
$$

by Lemma 6 the equilibrium point $x_{\mathrm{SW}}$ is a repeller. By Lemma 7 there exist two minimal period-two solutions. Let $g(x, y)=f(y, x)$ and $h(x, y)=f(f(y, x), y)$. Then period-two curves, that is, the curves of which the intersection is a period-two solution, are given by

$$
\mathcal{C}_{\tilde{F}}:=\{(x, y): g(x, y)=x\}, \quad \mathcal{C}_{\tilde{G}}:=\{(x, y): h(x, y)=y\} .
$$

Taking derivatives of $g(x, y)=x$ and $h(x, y)=y$ with respect to $x$ we get

$$
y_{\tilde{F}}^{\prime}(x)=\frac{1-g_{x}^{\prime}(x, y)}{g_{y}^{\prime}(x, y)}, \quad y_{\tilde{G}}^{\prime}(x)=\frac{h_{x}^{\prime}(x, y)}{1-h_{y}^{\prime}(x, y)}
$$


One can see that

$$
\begin{aligned}
y_{\tilde{F}}^{\prime}(\phi)-y_{\tilde{G}}^{\prime}(\phi) & =\frac{1-g_{x}^{\prime}(\phi, \psi)}{g_{y}^{\prime}(\phi, \psi)}-\frac{h_{x}^{\prime}(\phi, \psi)}{1-h_{y}^{\prime}(\phi, \psi)} \\
& =\frac{1-e_{1}}{f_{1}}-\frac{g_{1}}{1-h_{1}}=\frac{1-\left(e_{1}+h_{1}\right)+\left(e_{1} h_{1}-f_{1} g_{1}\right)}{f_{1}\left(1-h_{1}\right)} \\
& =\frac{p(1)}{f_{1}\left(1-h_{1}\right)}=\frac{\left(1-\mu_{1}\right)\left(1-\mu_{2}\right)}{f(1-h)},
\end{aligned}
$$

where $p(\mu)$ is the characteristic equation of the matrix

$$
J_{T^{2}}(\Phi, \Psi)=\left(\begin{array}{ll}
e_{1} & f_{1} \\
g_{1} & h_{1}
\end{array}\right) .
$$

By Lemma 7, suppose that $\left\{\phi_{1}, \psi_{1}\right\}$ is a minimal period-two solution such that $\left\{\phi_{1}, \psi_{1}\right\}$ are roots of multiplicity two of (12) and $\left\{\phi_{2}, \psi_{2}\right\}$ are roots of multiplicity one of (12). In view of Lemma 7 in [24] we have $y_{\tilde{F}}^{\prime}\left(\phi_{1}\right)-y_{\tilde{G}}^{\prime}\left(\phi_{1}\right)=0$ and $y_{\tilde{F}}^{\prime}\left(\phi_{2}\right)-y_{\tilde{G}}^{\prime}\left(\phi_{2}\right) \neq 0$, which implies that $\left\{\phi_{1}, \psi_{1}\right\}$ is non-hyperbolic. Since $\operatorname{det} J_{T}\left(\phi_{2}, \psi_{2}\right)>0$ and $\operatorname{tr} J_{T}\left(\phi_{2}, \psi_{2}\right)>0$ we see that $\left\{\phi_{2}, \psi_{2}\right\}$ is hyperbolic. Since $E_{S W}$ is a repeller it must be so that $\left\{\phi_{2}, \psi_{2}\right\}$ is a saddle point. If $\left\{\phi_{1}, \psi_{1}\right\}$ is non-hyperbolic of the stable type then the rest of the proof follows from Theorem 9.

4.2 Example 2: $x_{n+1}=A x_{n}+\frac{B x_{n-1}^{2}}{1+x_{n-1}^{2}}$

In this part we apply Theorems 5-10 to describe the global dynamics of difference equation in the title.

\subsubsection{The equilibrium points}

We consider the difference equation

$$
x_{n+1}=A x_{n}+\frac{B x_{n-1}^{2}}{1+x_{n-1}^{2}}, \quad n=0,1, \ldots
$$

where $A, B>0$ and the initial conditions $x_{-1}, x_{0}$ are non-negative. In view of the above restriction on the initial conditions of equation (14), the equilibrium points of equation (14) are the positive solutions of the equation

$$
\bar{x}=A \bar{x}+\frac{B \bar{x}^{2}}{1+\bar{x}^{2}}
$$

or equivalently

$$
(A-1) x^{3}+B x^{2}+(A-1) x=0 .
$$

Now we have the following result.

Lemma 9 The equilibrium points of equation (14) satisfy:

(i) If $A \geq 1$ or $B^{2}-4(A-1)^{2}<0$ then equation (14) has a unique equilibrium point $x_{0}=0$. 
(ii) If $A<1$ and $B^{2}-4(A-1)^{2}=0$ then equation (14) has two equilibrium points $x_{0}=0$, $x_{\mathrm{SW}}=\frac{B}{2(1-A)}$.

(iii) If $A<1$ and $B^{2}-4(A-1)^{2}>0$ then equation (14) has three equilibrium points $x_{0}=0, x_{\mathrm{SW}}=\frac{B-\sqrt{B^{2}-4(A-1)^{2}}}{2(1-A)}$, and $x_{\mathrm{NE}}=\frac{B+\sqrt{B^{2}-4(A-1)^{2}}}{2(1-A)}$.

Next, we investigate the stability of the equilibrium points of equation (14). Set

$$
f(u, v)=A u+\frac{B v^{2}}{1+v^{2}}
$$

and observe that $f_{u}(u, v)>0$ and $f_{v}(u, v)>0$. The next three lemmas are straightforward.

Lemma 10 Assume that $A, B>0$. Then the equilibrium point $x_{0}$ of equation (14) is locally asymptotically stable if $A<1$, non-hyperbolic if $A=1$, and a saddle point if $A>1$.

Lemma 11 Assume that $A<1$. If $B^{2}-4(A-1)^{2}>0$ then the equilibrium point $x_{\mathrm{NE}}$ of equation (14) is locally asymptotically stable and non-hyperbolic if $B^{2}-4(A-1)^{2}=0$.

Proof The proof follows from Theorem 1.1.1 in [2] and the fact that

$$
1-p-q=\frac{(1-A) \sqrt{B^{2}-4(A-1)^{2}}}{B} \text { and } p, q>0
$$

where $p=f_{u}\left(x_{\mathrm{NE}}, x_{\mathrm{NE}}\right)$ and $q=f_{u}\left(x_{\mathrm{NE}}, x_{\mathrm{NE}}\right)$.

Lemma 12 Assume that $A<1$. Then the equilibrium point $x_{\mathrm{SW}}$ is:

(i) a saddle point if $(A-1) \sqrt{B^{2}-4(A-1)^{2}}+2 A B>0$ and $B^{2}-4(A-1)^{2}>0$;

(ii) a repeller if $(A-1) \sqrt{B^{2}-4(A-1)^{2}}+2 A B<$ and $B^{2}-4(A-1)^{2}>0$;

(iii) a non-hyperbolic point if $B^{2}-4(A-1)^{2}=0$.

Proof The proof follows from Theorem 1.1.1 in [2] and the fact that

$$
1-p-q=\frac{(A-1) \sqrt{B^{2}-4(A-1)^{2}}}{B} \text { and } 1+p-q=\frac{(A-1) \sqrt{B^{2}-4(A-1)^{2}}+2 A B}{B}
$$

where $p=f_{u}\left(x_{\mathrm{SW}}, x_{\mathrm{SW}}\right)$ and $q=f_{u}\left(x_{\mathrm{SW}}, x_{\mathrm{SW}}\right)$.

\subsubsection{Period-two solutions}

Next, we investigate the existence and stability of the positive minimal period-two solutions of equation (14).

Let $\{\phi, \psi\}$ be a minimal period-two solution of equation (14). Then

$$
\phi=A \psi+\frac{B \phi^{2}}{1+\phi^{2}}, \quad \psi=A \phi+\frac{B \psi^{2}}{1+\psi^{2}}, \quad \phi \neq \psi
$$

which is true if and only if $\phi \neq \psi$ and

$$
\begin{aligned}
& -A \psi-A \psi \phi^{2}-B \phi^{2}+\phi^{3}+\phi=0, \\
& -A \psi^{2} \phi-A \phi-B \psi^{2}+\psi^{3}+\psi=0 .
\end{aligned}
$$


By eliminating $\psi$ from (16) and (17) we obtain

$$
\phi\left(A \phi^{2}+A+B \phi-\phi^{2}-1\right) \tilde{g}(\phi)=0,
$$

and by eliminating $\phi$ from (16) and (17) we obtain

$$
\psi\left(A \psi^{2}+A+B \psi-\psi^{2}-1\right) \tilde{g}(\psi)=0
$$

where

$$
\begin{aligned}
\tilde{g}(x)= & (A+1) x^{6}-2(A+1) B x^{5}+(A+1) x^{4}\left(A^{2}+B^{2}+2\right)-\left(A^{2}+3 A+2\right) B x^{3} \\
& +x^{2}\left(2 A^{3}+2 A^{2}+A B^{2}+A+1\right)-A(A+1) B x+A^{2}(A+1) .
\end{aligned}
$$

Since $(A-1) x^{2}+B x+A-1 \neq 0$ for any $x$ different from the equilibrium point, the minimal period-two solutions are solutions of the equation

$$
\tilde{g}(x)=0
$$

The proof of the following lemma is similar to the proof of Lemma 7, so we skip it.

Lemma 13 Let

$$
\begin{aligned}
\Delta= & A^{5}+20 A^{4}+8 A^{3} B^{2}+40 A^{3}-12 A^{2} B^{2}+40 A^{2}+4 A B^{4}-21 A B^{2}+20 A-B^{2}+4, \\
\Delta_{1}= & -\left(3 A^{2}-2 B^{2}+6\right), \\
\Delta_{2}= & \left(8 A^{7}+8 A^{6}+16 A^{5} B^{2}-32 A^{4} B^{2}+8 A^{3} B^{4}-45 A^{3} B^{2}\right. \\
& \left.-24 A^{3}+39 A^{2} B^{2}-24 A^{2}+8 A B^{4}-40 A B^{2}+16 A-4 B^{2}+16\right), \\
\Delta_{3}= & 4 A^{11}+192 A^{10}+212 A^{9} B^{2}-64 A^{9}+12 A^{8} B^{2}-704 A^{8}+264 A^{7} B^{4}+772 A^{7} B^{2} \\
& -384 A^{7}-108 A^{6} B^{4}+2,828 A^{6} B^{2}+896 A^{6}+148 A^{5} B^{6}+247 A^{5} B^{4}+1,516 A^{5} B^{2} \\
& +896 A^{5}+8 A^{4} B^{6}+237 A^{4} B^{4}-572 A^{4} B^{2}-384 A^{4}+32 A^{3} B^{8}+60 A^{3} B^{6} \\
& -363 A^{3} B^{4}+140 A^{3} B^{2}-704 A^{3}+32 A^{2} B^{8}-224 A^{2} B^{6}+247 A^{2} B^{4}+100 A^{2} B^{2} \\
& -64 A^{2}-40 A B^{6}+240 A B^{4}-336 A B^{2}+192 A+12 B^{4}-64 B^{2}+64, \\
\Delta_{4}= & \left(8-32 A^{2}+48 A^{4}-32 A^{6}+8 A^{8}-10 B^{2}-30 A B^{2}+57 A^{2} B^{2}\right. \\
& +8 A^{3} B^{2}+8 A^{4} B^{2}+70 A^{5} B^{2}-7 A^{6} B^{2}+2 B^{4}+26 A B^{4}-17 A^{2} B^{4}+8 A^{3} B^{4} \\
& \left.-15 A^{4} B^{4}-4 A B^{6}-4 A^{2} B^{6}\right), \\
\Delta_{5}= & \left(4 A^{4}-16 A^{3}+3 A^{2} B^{2}+24 A^{2}+2 A B^{2}-16 A-B^{2}+4\right) .
\end{aligned}
$$

Then the following holds:

(a) Consider equation (18). Then all its real roots are positive numbers. Furthermore, equation (14) has up to three minimal period-two solutions.

(b) If $\Delta_{i}>0$, for all $1 \leq i \leq 5$ and $\Delta>0$ then equation (18) has six real roots.

Consequently, equation (14) has three minimal period-two solutions. 
(c) If $\Delta_{i} \leq 0$ for some $1 \leq i \leq 4$ and $\Delta_{5}>0, \Delta>0$ then equation (18) has two distinct real roots and two pairs of conjugate imaginary roots. Consequently, equation (14) has one minimal period-two solutions.

(d) If $\Delta_{i}>0$ for all $1 \leq i \leq j-1$ and $\Delta_{i}<0$ for all $j \leq i \leq 4$ for some $1 \leq j \leq 5$ and $\Delta_{5}<0$, $\Delta>0$ then equation (18) has four distinct real roots and one pair of conjugate imaginary roots. Consequently, equation (14) has two minimal period-two solutions.

(e) If $\Delta_{i} \leq 0, \Delta_{i+1} \geq 0$ for some $1 \leq i \leq 3$ and $\Delta_{5}<0, \Delta>0$ then equation (18) has three pairs of conjugate imaginary roots. Consequently, equation (14) has no minimal period-two solution.

(f) Assume that $\Delta=0, \Delta_{3} \neq 0, \Delta_{5} \neq 0$.

(f.1) If $\left(\Delta_{1} \leq 0, \Delta_{2} \geq 0\right)$ or $\left(\Delta_{2} \leq 0, \Delta_{3}>0\right)$ then equation (18) has no real roots and has two distinct pairs of conjugate imaginary roots one of them is of multiplicity one and the other one of multiplicity two. Consequently, equation (14) has no minimal period-two solutions.

(f.2) If $\Delta_{1}>0, \Delta_{2}>0, \Delta_{3}>0$ then equation (18) has four distinct real roots, two of them are of multiplicity two and the other two of multiplicity one and has no conjugate imaginary roots. Consequently, equation (14) has two minimal period-two solutions.

\subsubsection{The global behavior}

In this section we describe the global behavior of equation (14). Equation (14) has three equilibrium points $\bar{x}_{0}, \bar{x}_{\mathrm{SW}}, \bar{x}_{\mathrm{NE}} \in I$ such that $0=\bar{x}_{0}<\bar{x}_{\mathrm{SW}}<\bar{x}_{\mathrm{NE}}$ where the equilibrium points $\bar{x}_{0}$ and $\bar{x}_{\mathrm{NE}}$ are locally asymptotically stable and $\bar{x}_{\mathrm{SW}}$ is unstable. One can see that all minimal period two solutions of (14) belong to $\operatorname{int}\left(Q_{2}\left(E_{\mathrm{SW}}\right) \cup Q_{4}\left(E_{\mathrm{SW}}\right)\right)$.

Let $u_{n+1}=A u_{n}+B$. By mathematical induction, it is easy to see that $x_{n} \leq u_{n}$ for $n>0$ if $x_{0} \leq u_{0}$. Since

$$
u_{n}=u_{0} A^{n}+\frac{B\left(1-A^{n}\right)}{1-A}, \quad A \neq 1
$$

and

$$
u_{n}=u_{0}+n B, \quad A=1 \text {, }
$$

we obtain $u_{n} \rightarrow \frac{B}{1-A}$ as $n \rightarrow \infty$ if $A<1$. Thus, we conclude that the interval $\left[0, \frac{B}{1-A}+\epsilon\right]$ where $\epsilon>0$ attracts all solutions, when $A<1$.

Lemma 14 If $A \geq 1$ then there exists a unique equilibrium point $x_{0}=0$ and every solution $\left\{x_{n}\right\}$ satisfies

$$
\lim _{n \rightarrow \infty} x_{n}=\infty
$$

Proof By using the difference inequalities method [25], the proof follows from the fact that $x_{n} \geq v_{n}$ for $n>0$ where $x_{0}=v_{0}$ and $v_{n}=A v_{n-1}$ for $n>1$.

Theorem 12 Assume that $A<1$ and $B^{2}-4(A-1)^{2}>0$. Then the following holds: 
(i) If $\Delta_{i} \leq 0, \Delta_{i+1} \geq 0$ for some $1 \leq i \leq 3$ and $\Delta_{5}<0, \Delta>0$ then equation (14) has three equilibrium point such that $0 \leq \bar{x}_{0}<\bar{x}_{\mathrm{SW}}<\bar{x}_{\mathrm{NE}}$ where $x_{0}$ and $x_{\mathrm{NE}}$ are locally asymptotically stable and $x_{\mathrm{SW}}$ is a saddle point and has no period-two solution. The global behavior of equation (14) is described by Theorem 6. For example, this happens for $A=0.3$ and $B=2.0$.

(ii) If $\Delta_{i} \leq 0$ for some $1 \leq i \leq 4$ and $\Delta_{5}>0, \Delta>0$ then equation (14) has three equilibrium points such that $0 \leq \bar{x}_{0}<\bar{x}_{\mathrm{SW}}<\bar{x}_{\mathrm{NE}}$ where $x_{0}$ and $x_{\mathrm{NE}}$ are locally asymptotically stable and $x_{\mathrm{SW}}$ is a repeller and one period-two solution $\left\{\phi_{1}, \psi_{1}\right\}$ which is a saddle point. The global behavior of equation (14) is described by Theorem 7. For example, this happens for $A=0.1$ and $B=1.9$.

(iii) If $\Delta_{i}>0$, for all $1 \leq i \leq 5$ and $\Delta>0$ then equation (14) has three equilibrium points such that $0 \leq \bar{x}_{0}<\bar{x}_{\mathrm{SW}}<\bar{x}_{\mathrm{NE}}$ where $x_{0}$ and $x_{\mathrm{NE}}$ are locally asymptotically stable and $x_{\mathrm{SW}}$ is a repeller and three minimal period-two solutions $\left\{\phi_{1}, \psi_{1}\right\},\left\{\phi_{2}, \psi_{2}\right\}$, and $\left\{\phi_{3}, \psi_{3}\right\}$ such that $\left(\phi_{1}, \psi_{1}\right) \preceq_{\text {ne }}\left(\phi_{2}, \psi_{2}\right) \preceq_{\text {ne }}\left(\phi_{3}, \psi_{3}\right)$ where $\left\{\phi_{1}, \psi_{1}\right\}$, and $\left\{\phi_{3}, \psi_{3}\right\}$ are saddle points and $\left\{\phi_{2}, \psi_{2}\right\}$ is locally asymptotically stable. The global behavior of equation (14) is described by Theorem 8. For example, this happens for $A=0.1$ and $B=2.0$.

(iv) If $\Delta=0$ and $\Delta_{5}>0$ and $\Delta_{1}>0, \Delta_{2}>0, \Delta_{3}>0$ then equation (14) has three equilibrium points such that $0 \leq \bar{x}_{0}<\bar{x}_{\mathrm{SW}}<\bar{x}_{\mathrm{NE}}$ where $x_{0}$ and $x_{\mathrm{NE}}$ are locally asymptotically stable and $x_{\mathrm{SW}}$ is a repeller and two minimal period-two solutions $\left\{\phi_{1}, \psi_{1}\right\}$ and $\left\{\phi_{2}, \psi_{2}\right\}$ such that $\left(\phi_{1}, \psi_{1}\right) \preceq_{\text {ne }}\left(\phi_{2}, \psi_{2}\right)$ where either $\left\{\phi_{1}, \psi_{1}\right\}$ is non-hyperbolic and $\left\{\phi_{2}, \psi_{2}\right\}$ is a saddle point or $\left\{\phi_{1}, \psi_{1}\right\}$ is a saddle point and $\left\{\phi_{2}, \psi_{2}\right\}$ is non-hyperbolic. If a period-two solution which is non-hyperbolic is of stable type then the global behavior of equation (14) is described by Theorems 8 and 9. For example, this happens for $A=0.1$ and $B=1.97282$.

Remark 2 Similarly as in the previous two examples, one can see that the difference equation

$$
x_{n+1}=\frac{A x_{n}}{1+x_{n}}+\frac{B x_{n-1}^{2}}{1+x_{n-1}^{2}}, \quad A, B>0, x_{-1}, x_{0} \geq 0, n=0,1, \ldots
$$

has three equilibrium points $\bar{x}_{0}, \bar{x}_{\mathrm{SW}}, \bar{x}_{\mathrm{NE}} \in[0, \infty)$ such that $0=\bar{x}_{0}<\bar{x}_{\mathrm{SW}}<\bar{x}_{\mathrm{NE}}$ where the equilibrium points $\bar{x}_{0}$ and $\bar{x}_{\mathrm{NE}}$ are locally asymptotically stable and $\bar{x}_{\mathrm{SW}}$ is unstable and $x_{n}<A+B$ for all $n \geq 1$. Further, one can see that all its minimal period two solutions belong to $\operatorname{int}\left(Q_{2}\left(E_{\mathrm{SW}}\right) \cup Q_{4}\left(E_{\mathrm{SW}}\right)\right)$. It has up to three minimal period-two solutions and the global behavior can be described by Theorem 12, where the determinants $\Delta_{i}$ will have different values which depend of the discrimination matrix.

All figures are created by Dynamica 4 [26].

Competing interests

The authors declare that they have no competing interests.

Authors' contributions

All authors contributed equally to this work. They all read and approved the final version of the manuscript.

Author details

1 Department of Mathematics, University of Rhode Island, Kingston, RI 02881-0816, USA. ²Department of Mathematics, University of Sarajevo, Sarajevo, Bosnia and Herzegovina. 


\section{Acknowledgements}

MRS Kulenović is supported in part by Maitland P Simmons Foundation. Esmir Pilav is supported in part by FMON of Bosnia and Herzegovina, number 05-39-3935-1/15.

Received: 3 February 2016 Accepted: 5 March 2016 Published online: 14 March 2016

\section{References}

1. Hautus, MLJ, Bolis, TS: Solution to Problem E2721. Am. Math. Mon. 86, 865-866 (1979)

2. Kulenović, MRS, Ladas, G: Dynamics of Second Order Rational Difference Equations with Open Problems and Conjectures. Chapman \& Hall/CRC, Boca Raton (2001)

3. Amleh, AM, Camouzis, E, Ladas, G: On the dynamics of a rational difference equation, part I. Int. J. Difference Equ. 3, 1-35 (2008)

4. Camouzis, E, Ladas, G: Dynamics of Third-Order Rational Difference Equations with Open Problems and Conjectures. Advances in Discrete Mathematics and Applications, vol. 5. Chapman \& Hall/CRC, Boca Raton (2008)

5. Brett, A, Kulenović, MRS: Basins of attraction of equilibrium points of monotone difference equations. Sarajevo J. Math. 5, 211-233 (2009)

6. Kulenović, MRS, Yakubu, A-A: Compensatory versus overcompensatory dynamics in density-dependent Leslie models. J. Differ. Equ. Appl. 10, 1251-1265 (2004)

7. Kulenović, MRS, Moranjkić, S, Nurkanović, Z: Global dynamics and bifurcation of a perturbed sigmoid Beverton-Holt difference equation. Math. Methods Appl. Sci. (2016). doi:10.1002/mma.3722

8. Robinson, C: Stability, Symbolic Dynamics, and Chaos. CRC Press, Boca Raton (1995)

9. Kulenović, MRS, Merino, O: Global bifurcation for competitive systems in the plane. Discrete Contin. Dyn. Syst., Ser. B 12, 133-149 (2009)

10. Hess, P: Periodic-Parabolic Boundary Value Problems and Positivity. Pitman Research Notes in Mathematics Series, vol. 247. Longman, Harlow (1991); copublished in the United States with Wiley, New York

11. de Mottoni, P, Schiaffino, A: Competition systems with periodic coefficients: a geometric approach. J. Math. Biol. 11, 319-335 (1981)

12. Hirsch, M, Smith, H: Monotone dynamical systems. In: Handbook of Differential Equations: Ordinary Differential Equations, vol. II, pp. 239-357. Elsevier, Amsterdam (2005)

13. Hirsch, M, Smith, HL: Monotone maps: a review. J. Differ. Equ. Appl. 11, 379-398 (2005)

14. Smith, HL: Planar competitive and cooperative difference equations. J. Differ. Equ. Appl. 3, 335-357 (1998)

15. Takáč, P: Asymptotic behavior of strongly monotone time-periodic dynamical processes with symmetry. J. Differ. Equ. 100(2), 355-378 (1992)

16. Takáč, P: Domains of attraction of generic $\omega$-limit sets for strongly monotone discrete-time semigroups. J. Reine Angew. Math. 423, 101-173 (1992)

17. Brett, A, Kulenović, MRS: Two species competitive model with the Allee effect. Adv. Differ. Equ. 2014, 307 (2014)

18. Kulenović, MRS, Merino, O: Competitive-exclusion versus competitive-coexistence for systems in the plane. Discrete Contin. Dyn. Syst., Ser. B 6, 1141-1156 (2006)

19. Kulenović, MRS, Merino, O: Invariant manifolds for competitive discrete systems in the plane. Int. J. Bifurc. Chaos Appl. Sci. Eng. 20(8), 2471-2486 (2010)

20. Smith, HL: Periodic solutions of periodic competitive and cooperative systems. SIAM J. Math. Anal. 17, 1289-1318 (1986)

21. Hadžiabdić, V, Kulenović, MRS, Pilav, E: Dynamics of a two-dimensional competitive system of rational difference equations with quadratic terms. Adv. Differ. Equ. 2013, 301 (2014)

22. Janson, S: Resultant and discriminant of polynomials. http://www2.math.uu.se/ svante/papers/ (2010)

23. Yang, L, Hou, X, Zeng, Z: Complete discrimination system for polynomials. Sci. China Ser. E 39(6), 628-646 (1996)

24. Basu, S, Merino, O: On the behavior of solutions of a system of difference equations. Commun. Appl. Nonlinear Anal. 16(1), 89-101 (2009)

25. Lakshmikantham, $\vee$, Trigiante, D: Theory of Difference Equations: Numerical Methods and Applications, 2 nd edn. Monographs and Textbooks in Pure and Applied Mathematics, vol. 251. Dekker, New York (2002)

26. Kulenović, MRS, Merino, O: Discrete Dynamical Systems and Difference Equations with Mathematica. Chapman \& Hall/CRC, Boca Raton (2002)

\section{Submit your manuscript to a SpringerOpen ${ }^{\circ}$ journal and benefit from:}

- Convenient online submission

- Rigorous peer review

- Immediate publication on acceptance

- Open access: articles freely available online

- High visibility within the field

- Retaining the copyright to your article

Submit your next manuscript at $>$ springeropen.com 